\title{
Pertussis in Individuals with Co-morbidities: A Systematic Review
}

\author{
Denis Macina · Keith E. Evans
}

Received: March 15, 2021 / Accepted: May 24, 2021 / Published online: June 12, 2021

(C) The Author(s) 2021

\begin{abstract}
Pertussis is a highly contagious disease of the respiratory tract caused by Bordetella pertussis. Although the burden of pertussis is highest in children, available data suggests that pertussis in the elderly and those with underlying chronic conditions or illnesses can result in significant morbidity, mortality and costs. We undertook a comprehensive review to assess the association between pertussis and chronic conditions/illnesses. A search was undertaken on 17 June 2019 across EMBASE, Medline and BIOSIS. Citations were limited to those in English, in humans and published since 1 January 1990. There were 1179 papers identified with an additional 70 identified through a review of the reference lists. Of these, 34 met the inclusion criteria. Papers included were categorised in groups, those which reported: associations between prior pertussis and subsequent chronic conditions or illnesses; a link between chronic conditions/illnesses and subsequent risk of pertussis; and those which reported on the effect of the chronic conditions/illnesses on
\end{abstract}

D. Macina $(\square)$

Vaccines Epidemiology and Modelling, Sanofi Pasteur, 14 Espace Henry Vallée, 69007 Lyon, France e-mail: Denis.Macina@sanofi.com

K. E. Evans

inScience Communications, Chowley Oak Business Park, Chowley Oak Lane, Tattenhall, Cheshire, UK pertussis complications or exacerbations. Pertussis appears to increase the likelihood of developing some chronic conditions/illnesses, but also appears to decrease the likelihood of developing some haematological cancers. There were several chronic conditions/illnesses where the study results were mixed, and several studies that found no association with previous pertussis. There were also studies which showed that having some comorbid health condition(s) might increase the risk of developing pertussis. Three studies showed pertussis can lead to increased exacerbations of chronic conditions/illnesses and associated hospitalisations, although one study showed it reduced the effects of chronic bronchitis. Previous pertussis appears to contribute to the increased likelihood of developing some respiratory conditions like asthma, and conversely those with asthma or COPD are at increased risk of severe pertussis requiring further intervention. Further research is required to confirm or disprove these associations, and to characterise the pathophysiological mechanisms behind the potential associations with pertussis.

\section{PLAIN LANGUAGE SUMMARY}

Pertussis, or whooping cough as it is more commonly known, is a respiratory disease that 
mainly affects young children, although it can be caught at any age. An increasing number of cases are being identified in older adults. This is concerning since older people typically have other underlying health conditions that can increase the risk of severe outcomes leading to increased mortality. We assessed 34 published studies that examined the link between whooping cough and some health conditions. Several studies found that prior whooping cough was more likely in those with an underlying health condition, and this was particularly true in those with respiratory conditions like asthma and chronic obstructive pulmonary disease, whilst there were also studies which showed that having some health condition(s) might increase the risk of developing severe whooping cough which might require medical attention or hospitalisation. There was also some evidence that previous whooping cough might be protective against some blood cancers. Whooping cough was shown to exacerbate several underlying health conditions, although a single study found that it may reduce the risk of chronic bronchitis exacerbations. More research is required to corroborate these findings.

Keywords: Comorbidity; Infectious disease; Pertussis; Systematic review; Underlying condition; Chronic illness

\section{Key Summary Points}

Pertussis is increasingly becoming a disease affecting an older group of people rather than young children.

A significant percentage of older people infected with Bordetella pertussis experience considerable morbidity, mortality and costs.

We reviewed 34 studies which considered the association between pertussis and underlying conditions/illnesses.
Previous infection with pertussis appears to contribute to the increased likelihood of developing some respiratory conditions like asthma and COPD, and conversely those with asthma or COPD are at increased risk of pertussis infection and for severe pertussis and/or asthma or COPD exacerbations.

Further research is required to confirm or disprove these associations, and to characterise the pathophysiological mechanisms behind the potential associations.

\section{DIGITAL FEATURES}

This article is published with digital features, including a plain language summary, to facilitate understanding of the article. To view digital features for this article go to https://doi.org/10. 6084/m9.figshare.14627340.

\section{INTRODUCTION}

The World Health Organization (WHO) describes pertussis (or whooping cough) as 'a highly contagious disease of the respiratory tract caused by Bordetella pertussis', an exclusive human pathogen [1]. Although predominantly a paediatric disease, adolescents and adults can also contract the disease. In children the disease is characterised by paroxysmal cough followed by inspiratory 'whoop' and post-tussive vomiting, though the clinical course may be influenced by a number of factors including vaccination history and age. Adolescents and adults often have atypical symptoms, and may only present with a persistent prolonged cough illness. The WHO estimates that there were 151,074 cases worldwide in 2018, and up to 89,000 deaths have been reported in previous years (2008) [1]. Currently, vaccination remains the best available strategy to combat pertussis [2].

Infection with pertussis has been shown to have immunodulatory effects (with recent 
research focusing on the role of adenylate cyclase toxin [CyaA] and type III secretion system [TTSS] effector proteins) [3-8] that may impact the pathogenesis of underlying chronic conditions/illnesses, particularly with regards to mental health disorders and chronic inflammatory conditions [9]. There is evidence that B. pertussis infection can have an effect on asthmatic response [10], respiratory conditions $[6,11]$ and other atopic conditions [12].

Pertussis in patients with underlying chronic conditions such as asthma or chronic obstructive pulmonary disease can be associated with complications $[13,14]$ that can lead to increased healthcare costs [15]. In addition, pertussis may play a role in the aetiology of other diseases [16]. A recent estimate suggested that $60 \%$ of older people in Europe have at least two chronic conditions/illnesses [17]. Older people are often at significant risk of infectious diseases and resulting complications for several reasons, including no previous or incomplete immunization, waned immunity and immunosenescence.

Pertussis is increasingly being shown to affect the older population. A recent US estimate suggested that $59 \%$ of all cases between 2006-2015 were among adolescents and adults [18], a trend which has been noted in other countries [19]. B. pertussis infection in the elderly in particular can result in considerable morbidity, mortality and costs [20-23]. Complications associated with pertussis in adolescents and adults are not infrequent, and include urinary incontinence, rib fracture, pneumothorax, inguinal hernia, aspiration, pneumonia, seizures and otitis media [24]. It can also have a significant negative impact upon quality of life [25]. To date there has been little published on the relationship between pertussis and chronic conditions/illnesses, with only one previous review which focussed mainly on respiratory conditions in 'at-risk' populations [26]. The objective of our review was to assess any association between pertussis and chronic conditions/illnesses.

\section{METHODS}

A search was undertaken on 17 June 2019 across three databases EMBASE, Medline and BIOSIS. The search strategy used the phrases "(pertussis OR whooping cough) AND (chronic obstructive pulmonary disease OR $\mathrm{ag}^{*} \mathrm{OR}$ comorbid* OR

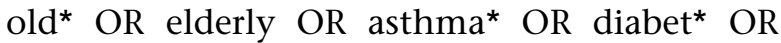
allerg* OR cardiovascular* OR frailty OR COPD OR chronic) AND (risk)". Citations were limited to those in English language, in humans and published since 1 January 1990. Papers were included if they met the following criteria: the full publication was in English and included subjects who had previously or have pertussis and at least one chronic condition/illness. Papers were excluded if they were reviews (i.e. contained no primary data but included if they contained data not reported elsewhere), reported data that was not categorised by chronic condition/illness, were vaccine trials (e.g. adverse events related to the vaccine) or singlesubject design (e.g. case studies). The reference lists of papers retrieved were checked for potentially useful studies not identified in the original search.

The two authors (DM and KE) assessed the studies independently and discussed any papers where there were disagreements as to their potential inclusion or exclusion. Studies which met the inclusion criteria were then entered into Microsoft Excel. Since the potential studies did not involve standardised study designs, and the interventions and comparators were not relevant, only participant data and outcomes data were entered, alongside the chronic condition/illness reported and the study strengths and weaknesses identified by the authors. Since most of the studies were observational studies of exposure, rather than an intervention, and in the absence of a formal and well-validated 'riskof-bias' tool for such studies [27], any formal analysis was not felt to be appropriate; however, each study was assessed against the Grading of Recommendations, Assessment, Development and Evaluation (GRADE) criteria prior to inclusion in our review [28] and the GRADE quality of evidence associated with each study is reported in Table 1 . Whilst the default for any 


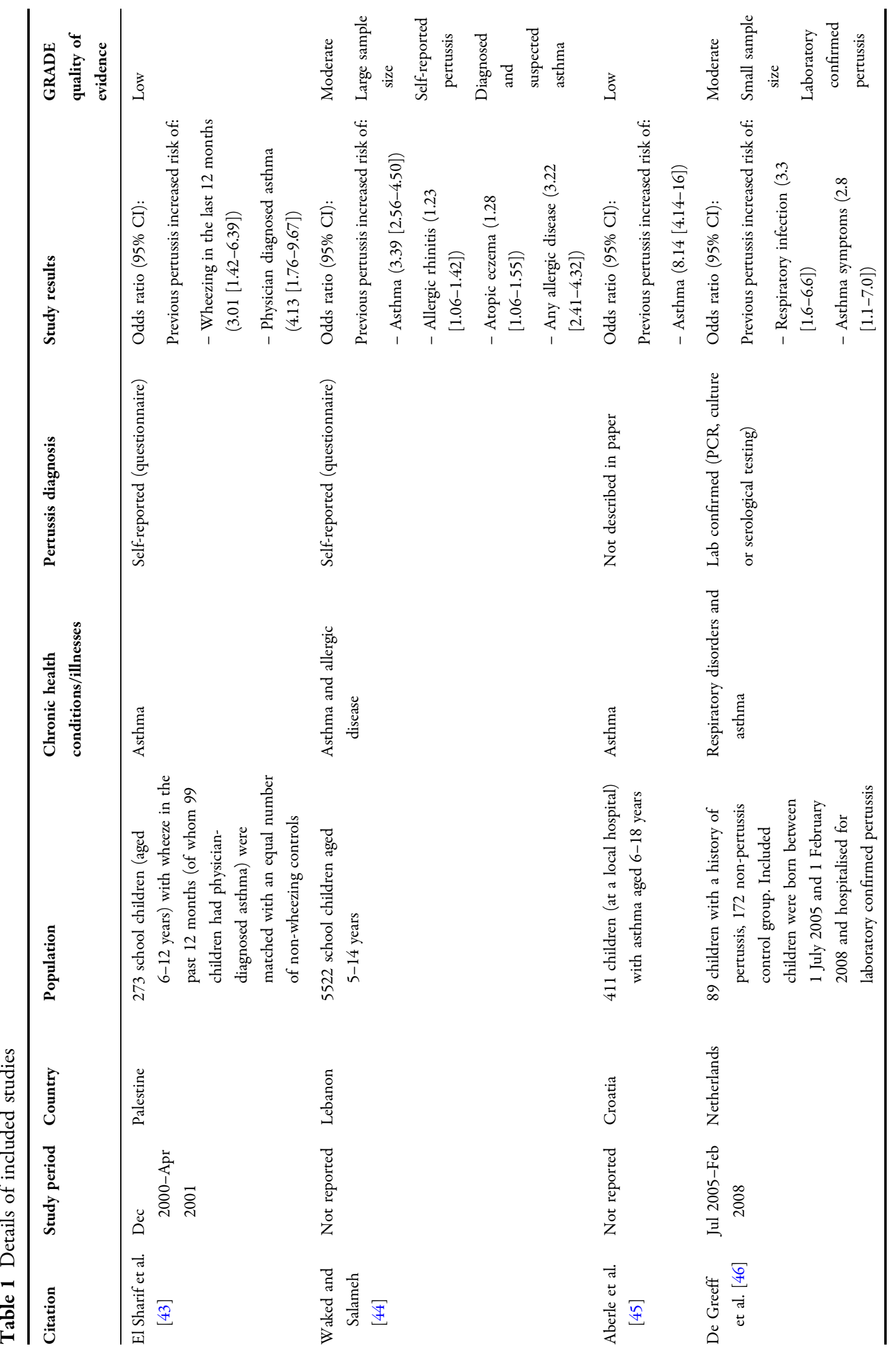




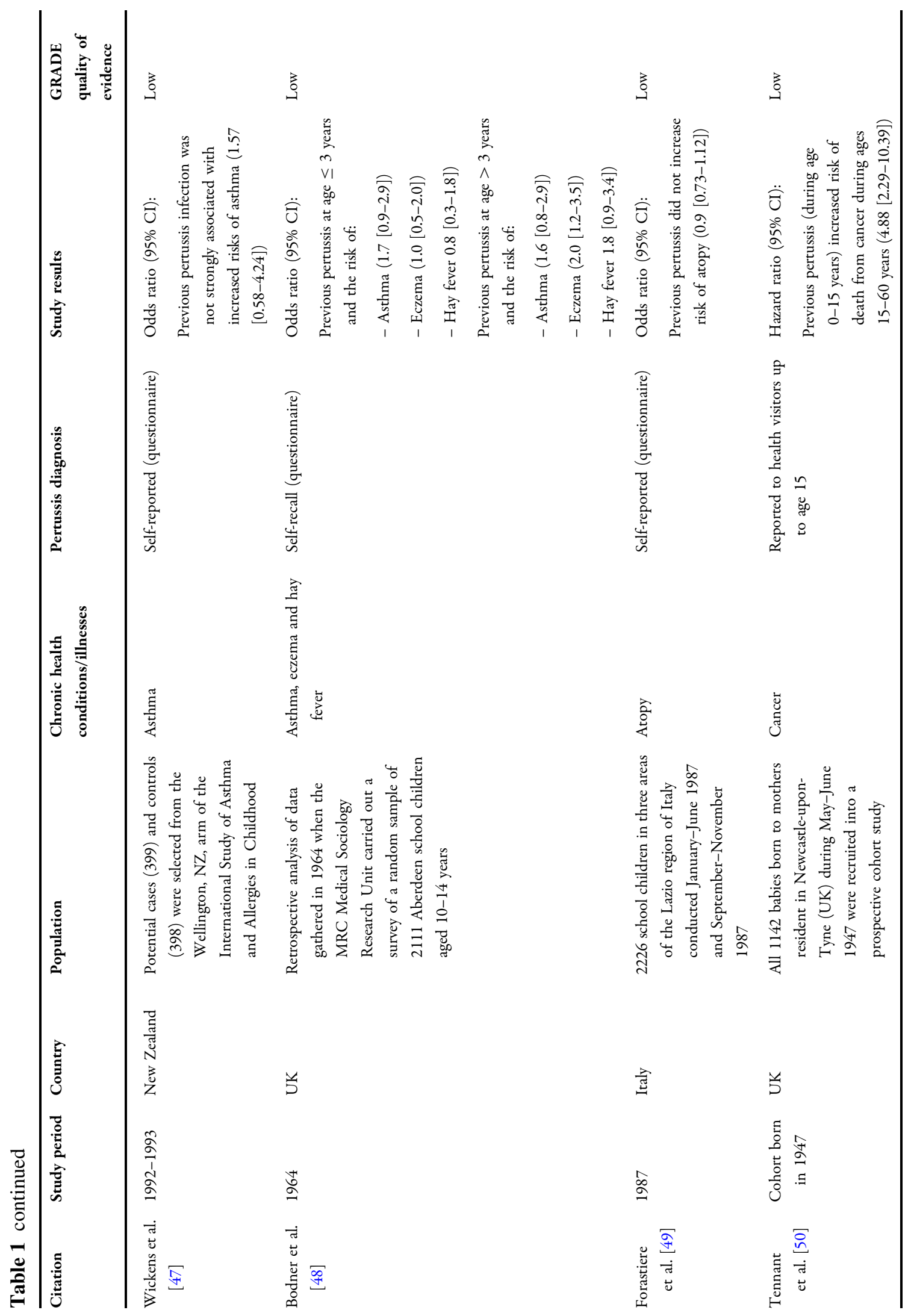




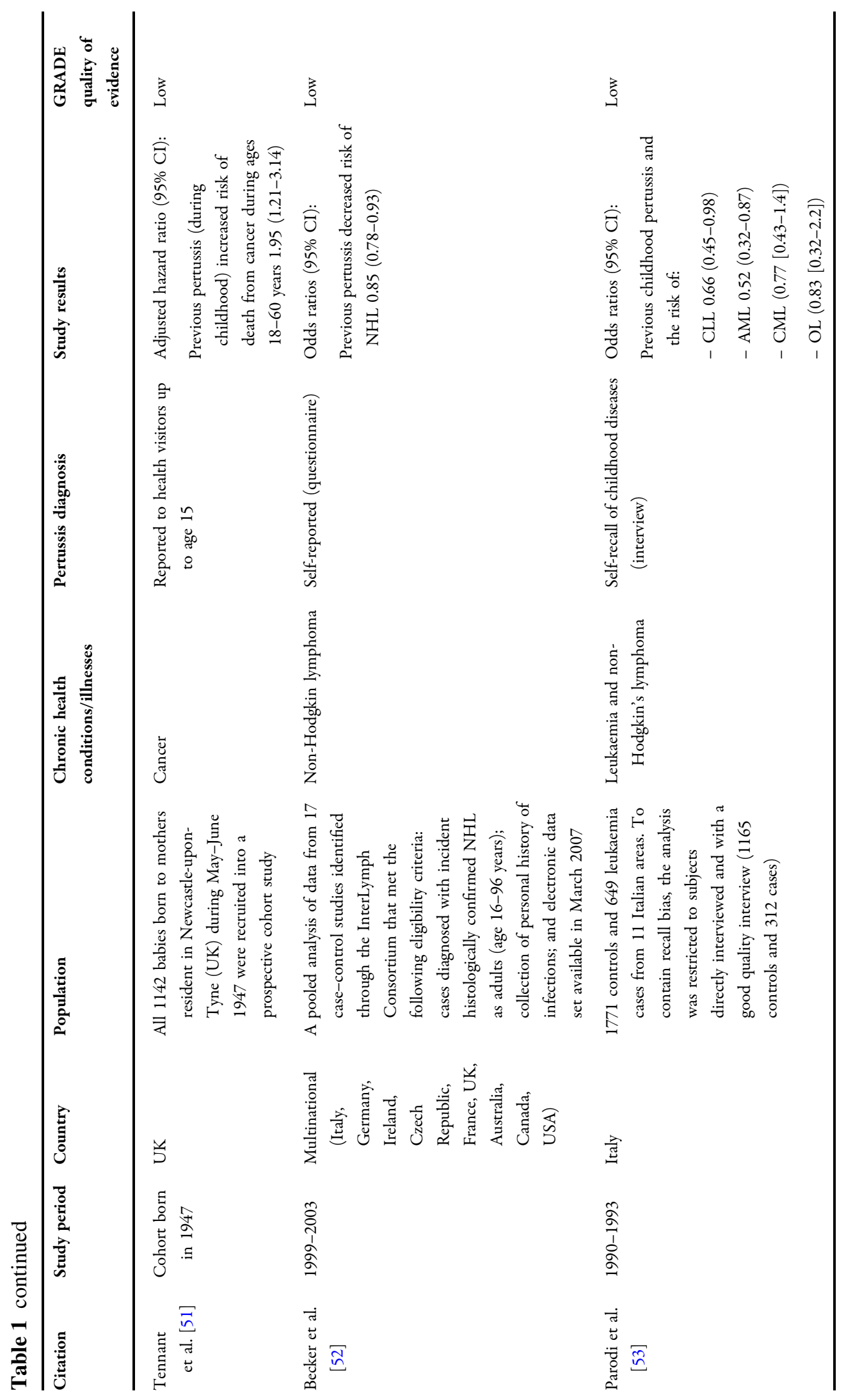




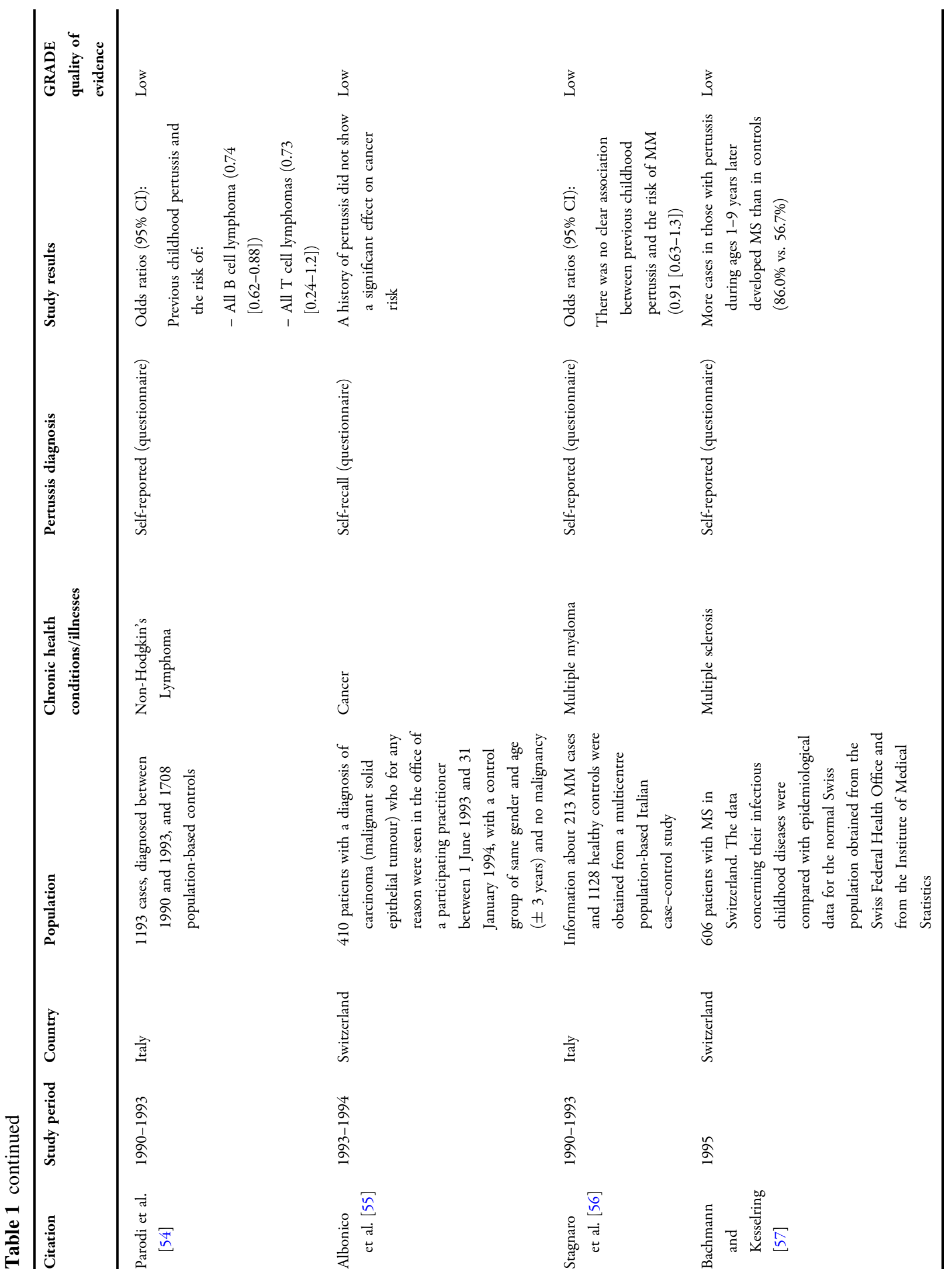




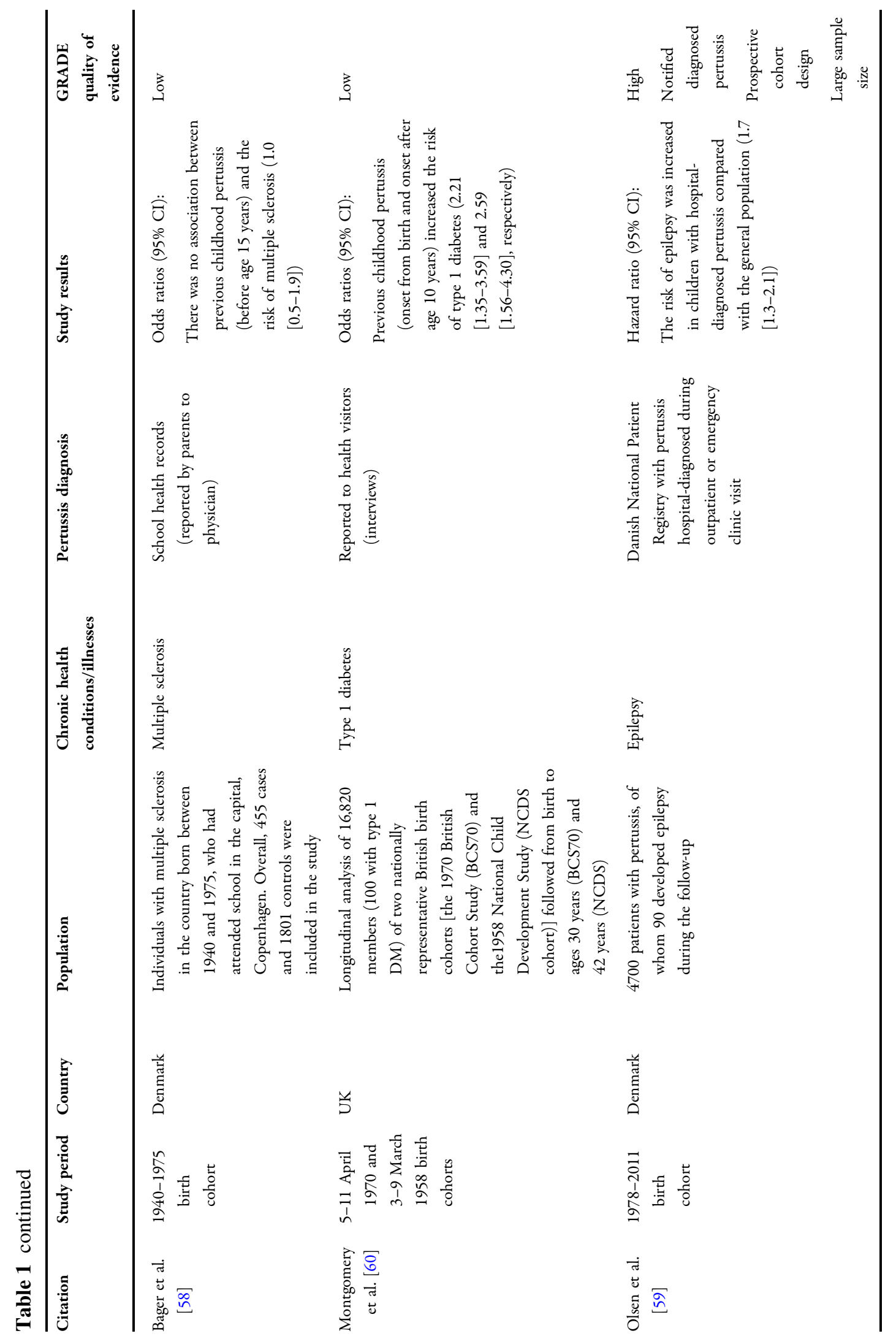




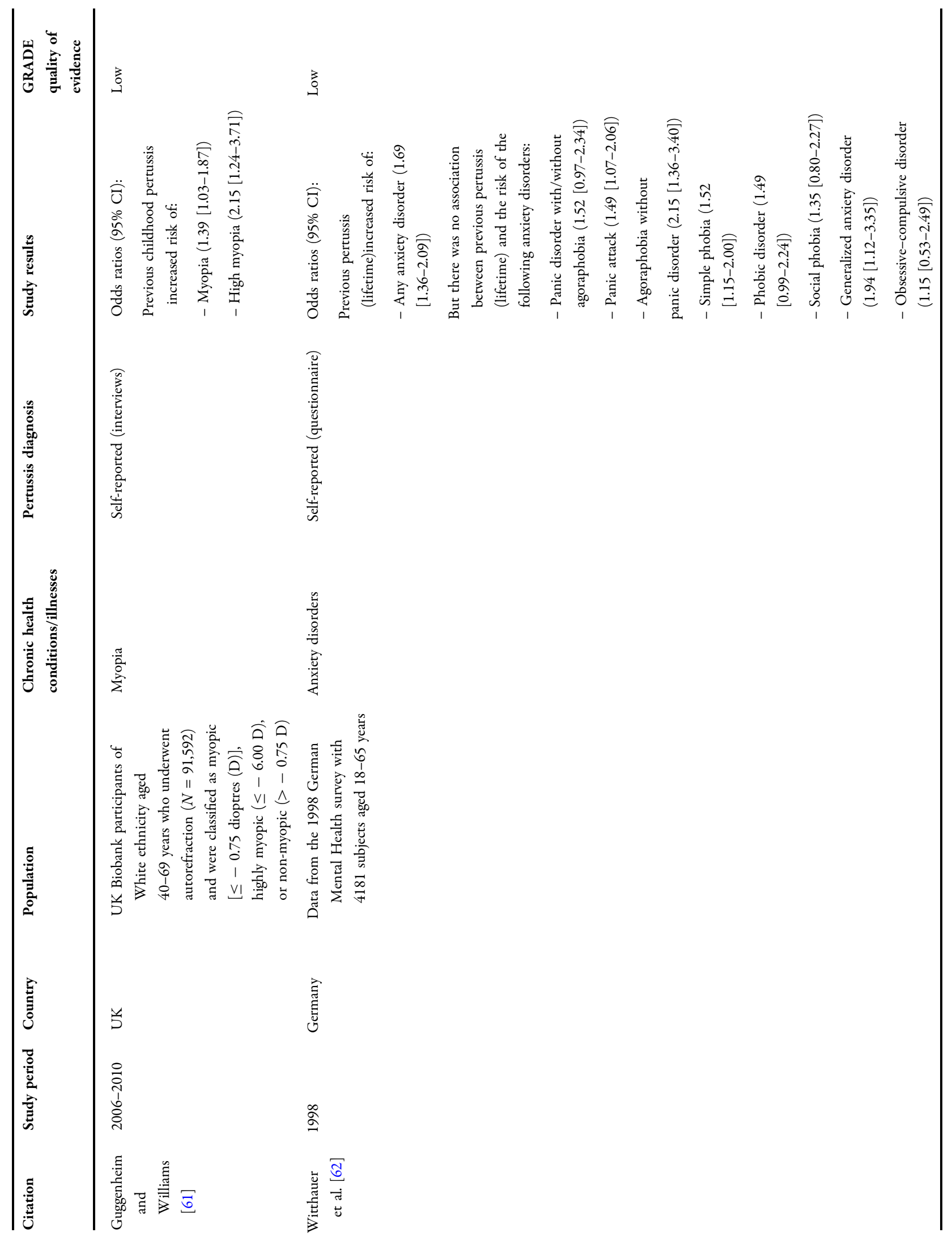




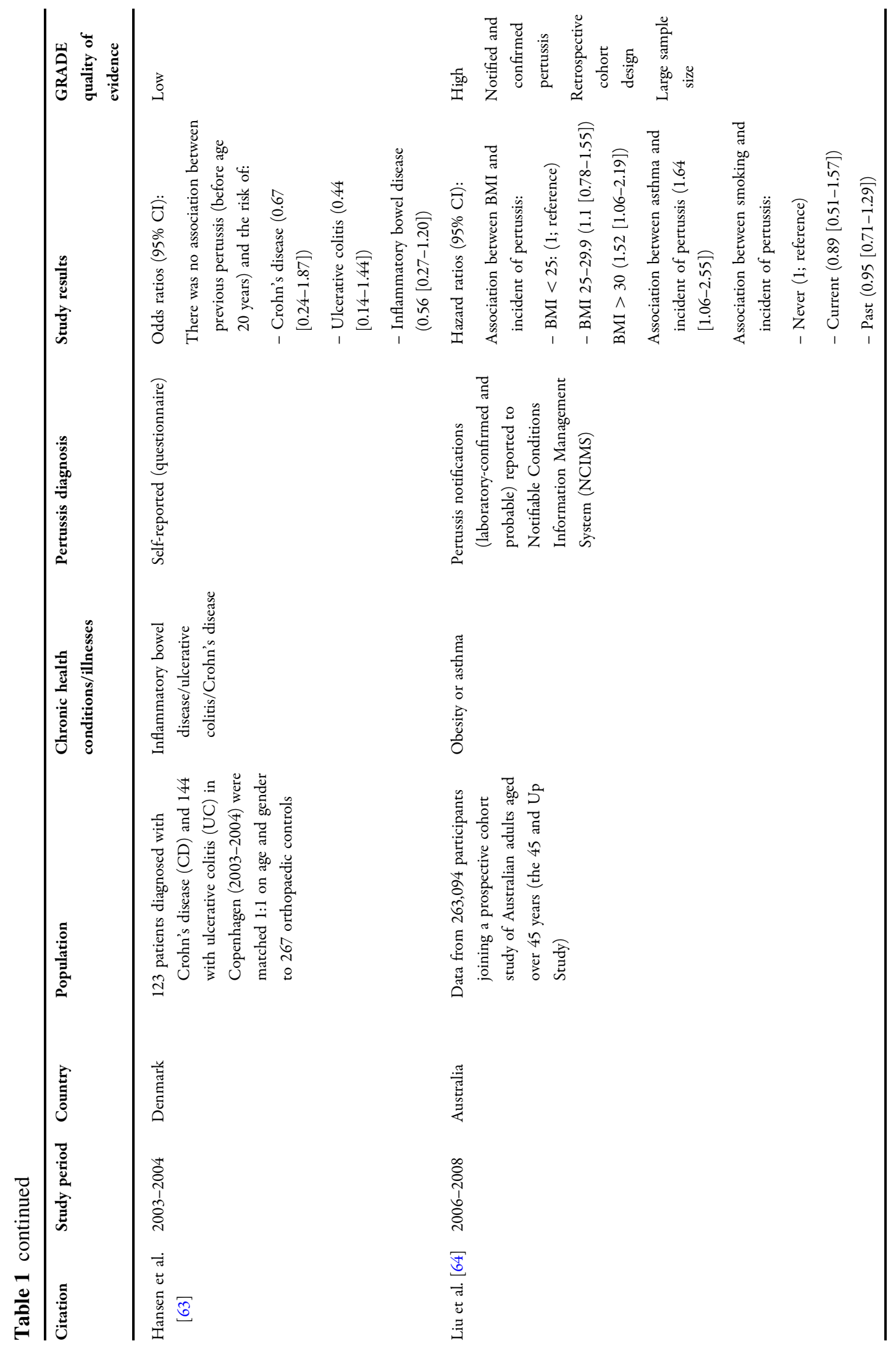




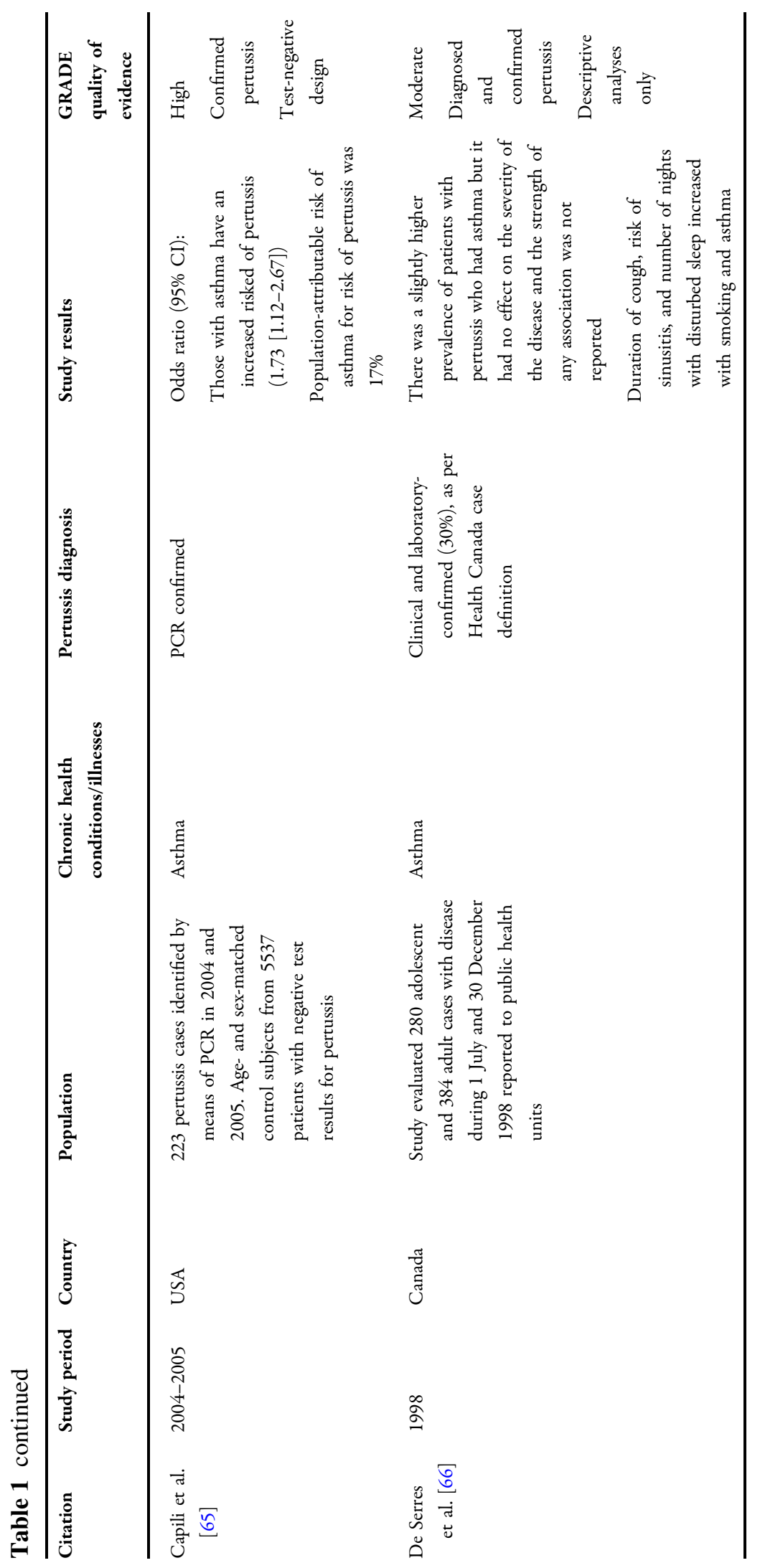




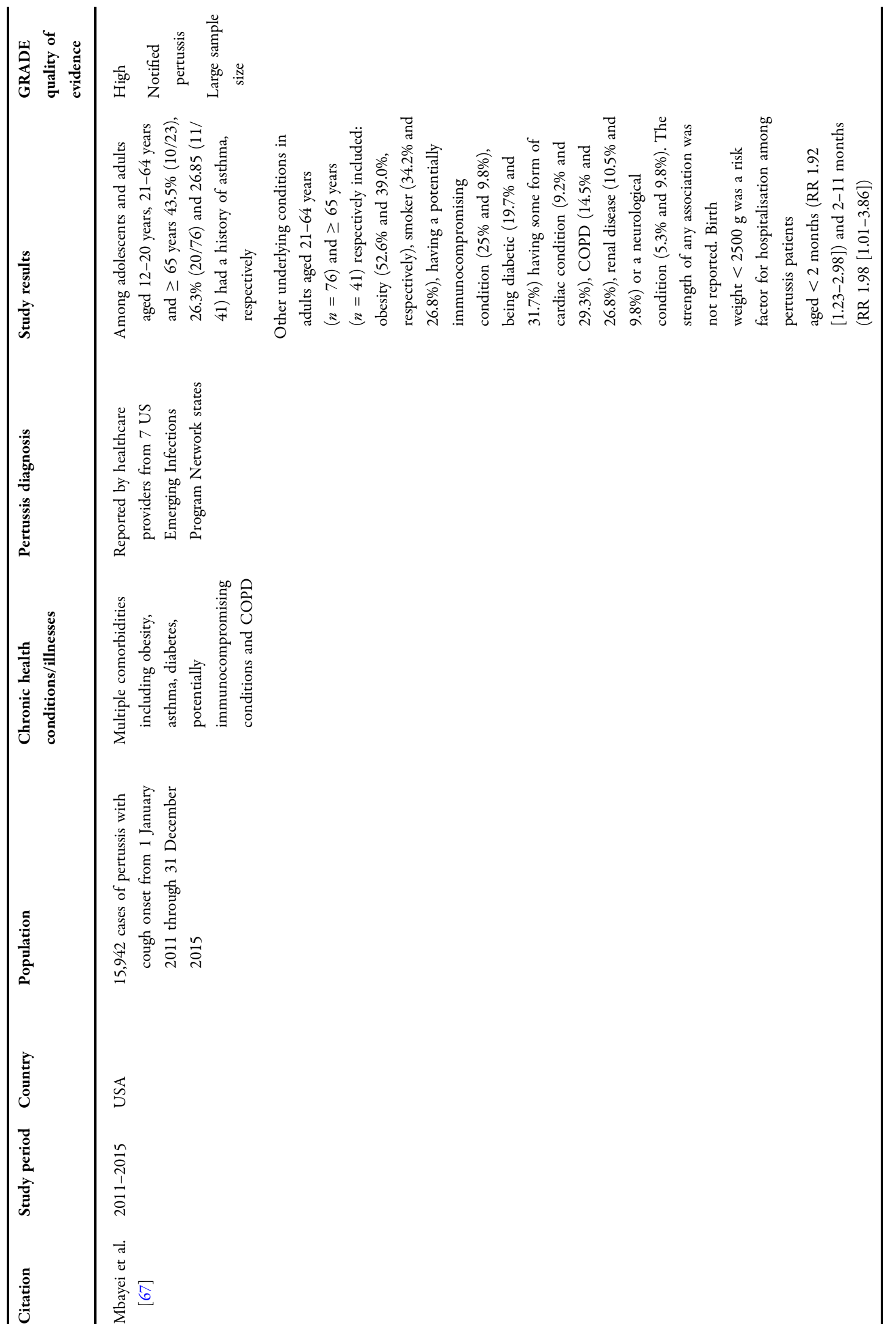




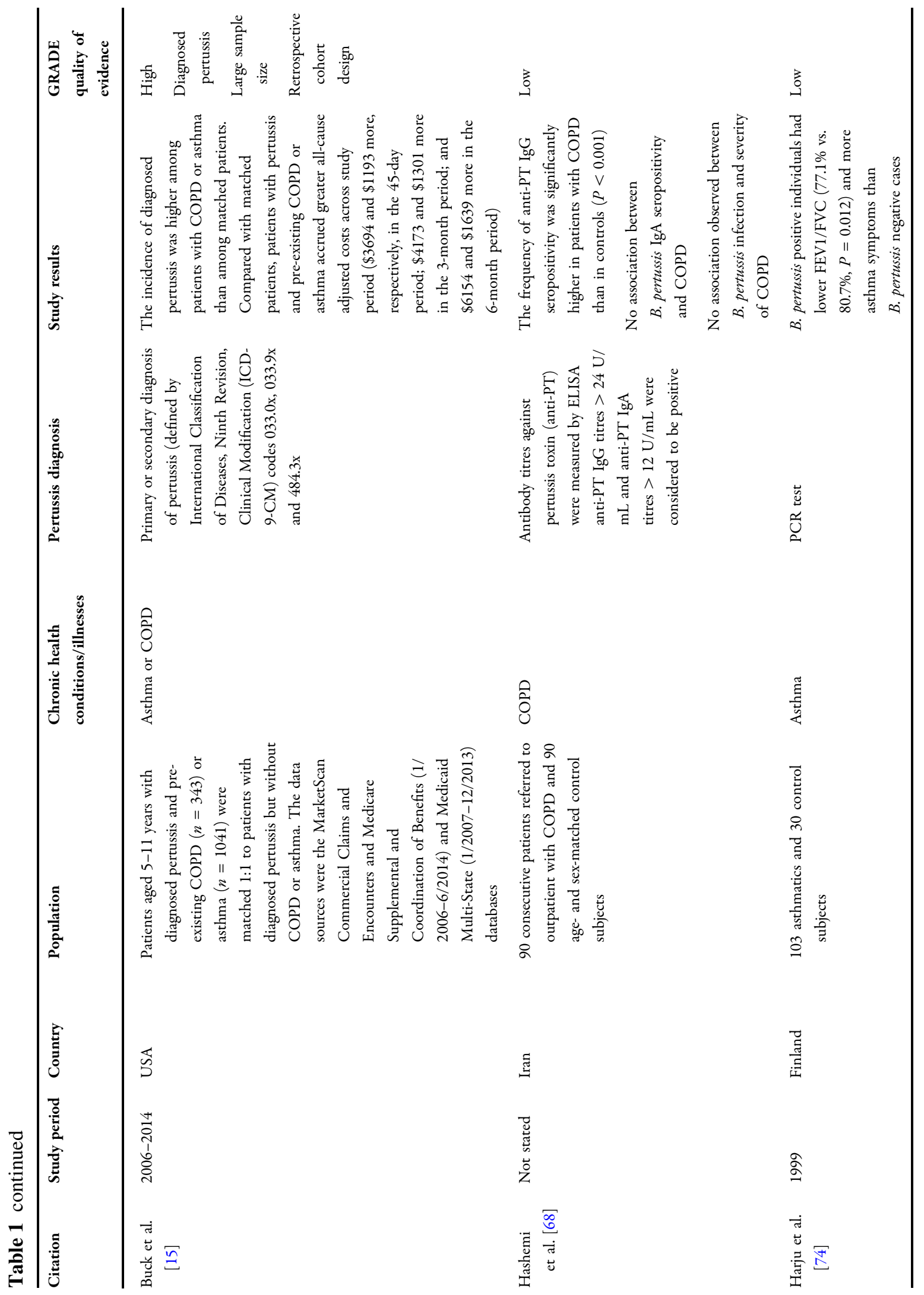




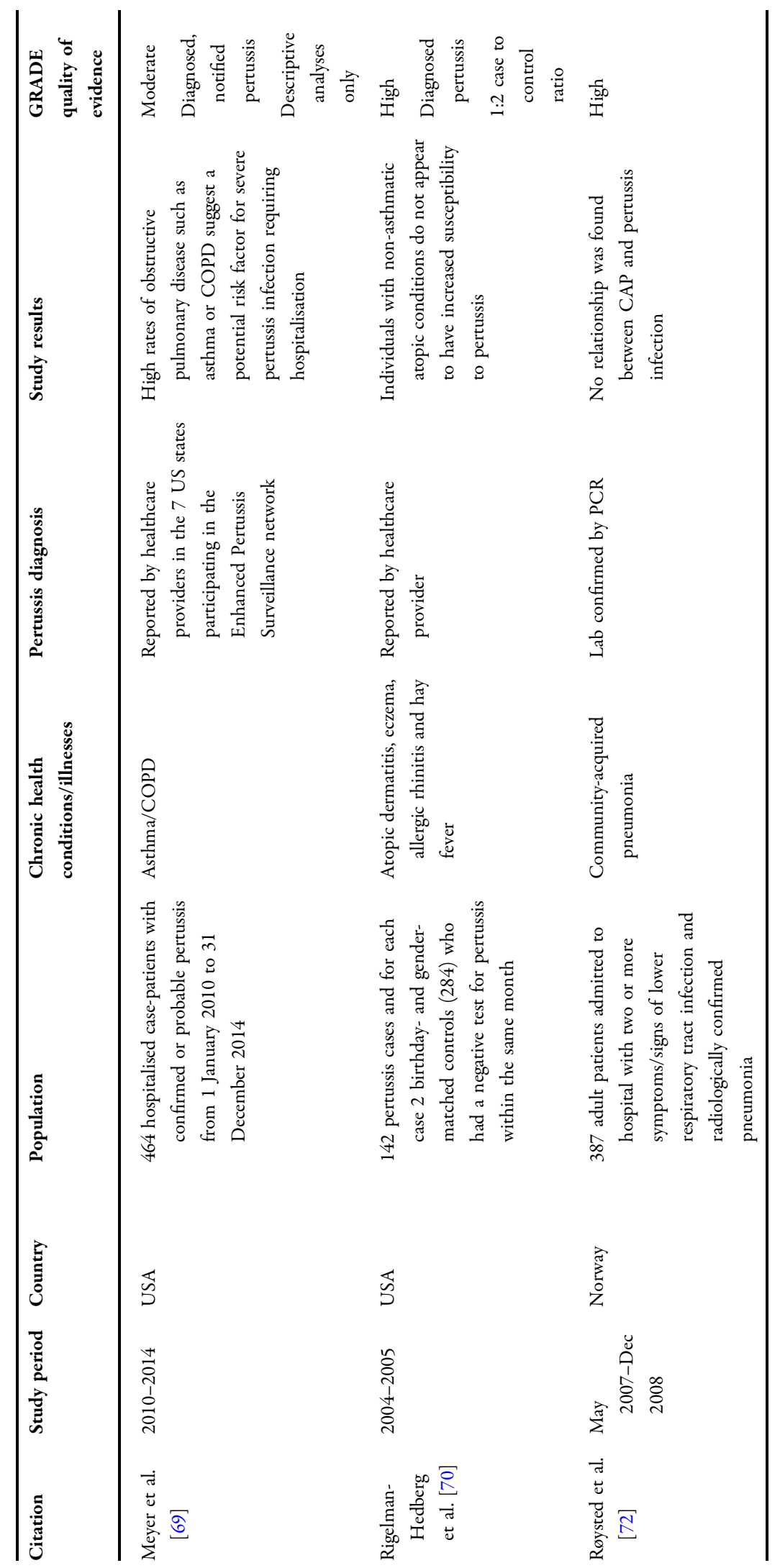




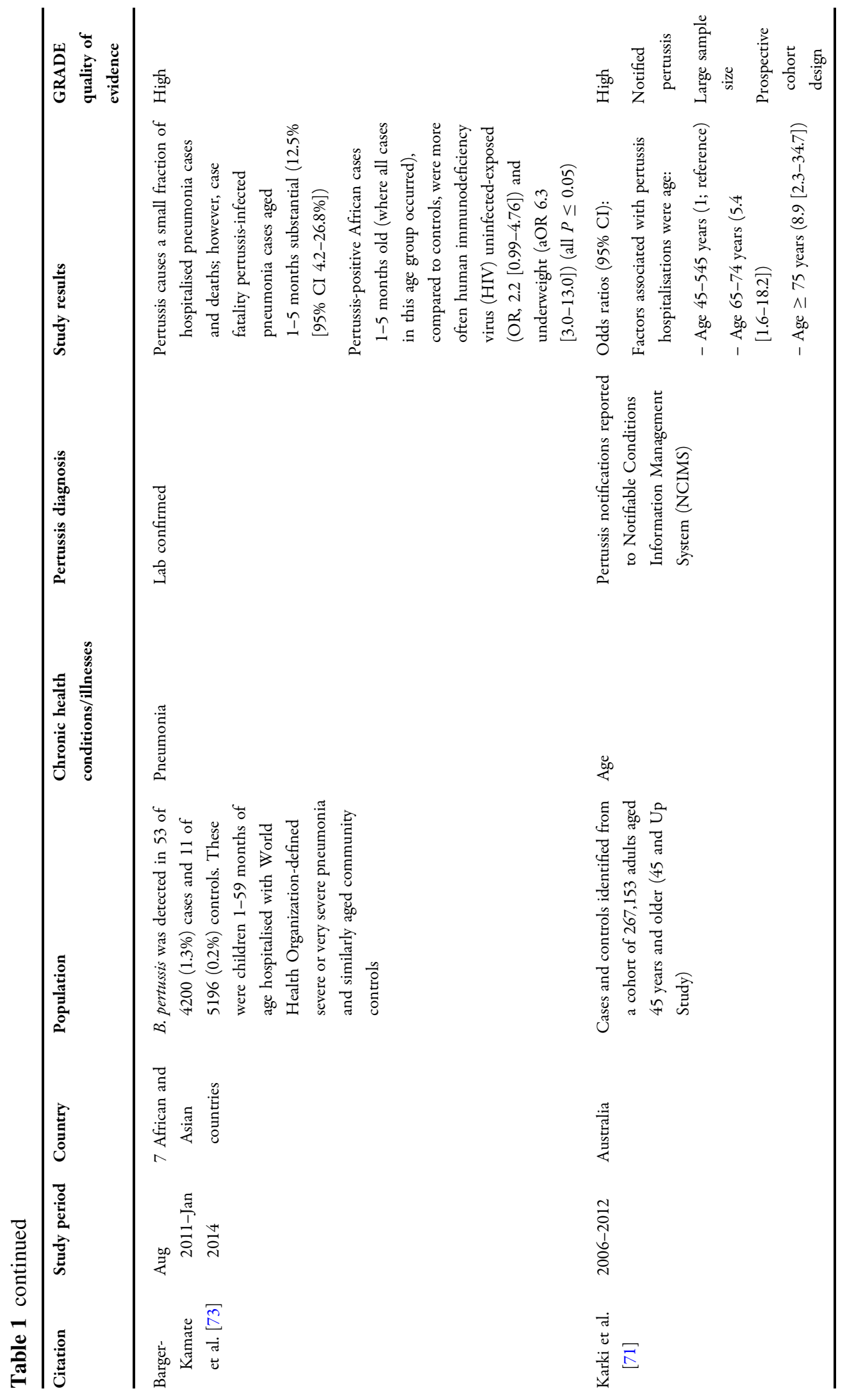




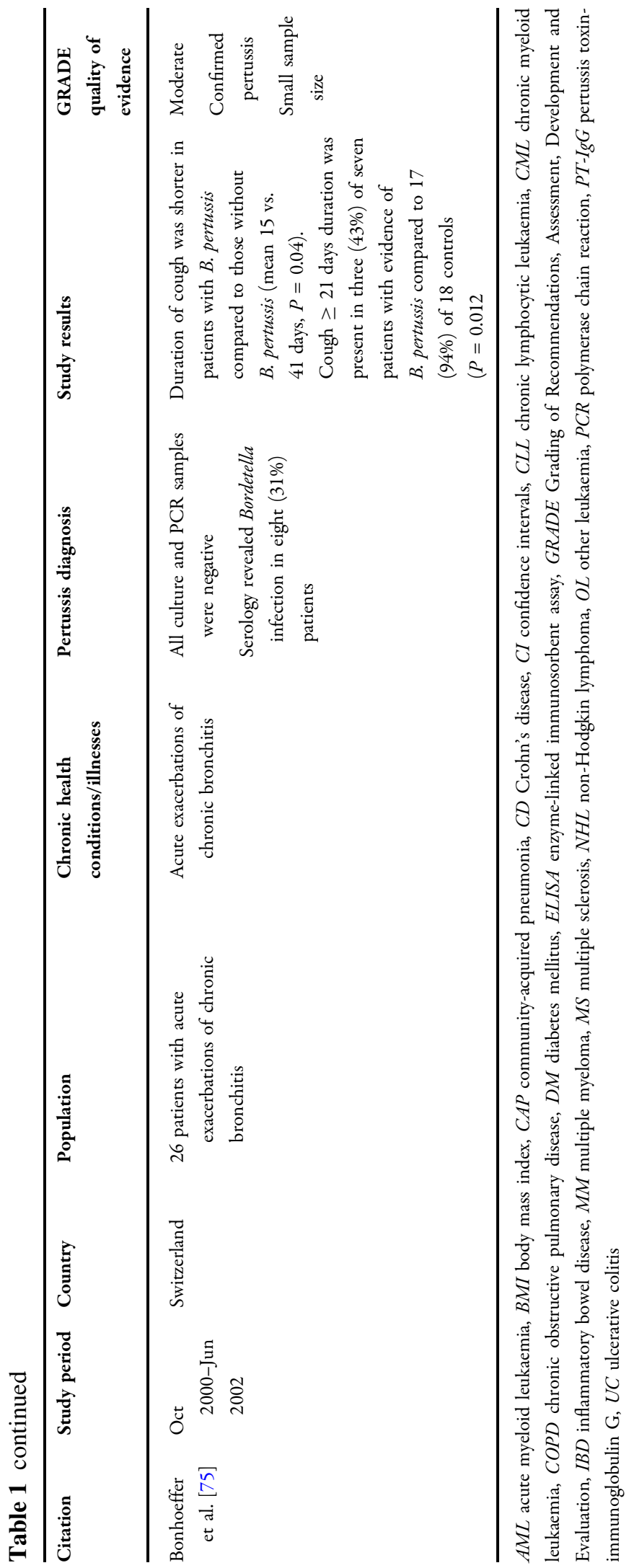


observational study is to describe it as 'low' quality, there is discretion within the GRADE system to upgrade the quality of evidence level based upon several factors. Where this has been done we have indicated the reasons for our upgrading in the table.

\section{Compliance with Ethics Guidelines}

This article is based on previously conducted studies and does not contain any new studies with human participants or animals performed by any of the authors.

\section{RESULTS}

There were 1179 papers identified through the search of databases with an additional 70 papers identified from other sources; after checking the abstract of the articles, 285 papers $(21 \%$ of the original search) were obtained for full assessment. The reasons for exclusion are summarised in Fig. 1. There was heterogeneity across the studies in terms of population assessed, study locations, identification of pertussis cases and study designs, which precluded meaningful statistical pooling/meta-analysis of the available data (Table 1). The available data are synthesized into a narrative summary focusing on general trends within selected groupings.

Overall, 34 papers met the inclusion criteria. These papers are summarised in three sections: those that reported associations between pertussis and subsequent chronic health conditions/illnesses, those which reported associations between underlying chronic conditions/illnesses and subsequent risk of pertussis, and those that reported on the effect of pertussis on the complications or severity of underlying chronic health conditions/illnesses. One of the methods used for the diagnosis of pertussis is to measure immunoglobulin $\mathrm{M}$ (IgM), immunoglobulin A (IgA) and immunoglobulin G (IgG) antibodies against B. pertussis by enzyme-linked immunosorbent assays (ELISA) [29], which are faster and more accurate than traditional bacterial agglutination tests [30] and immunofluorescence tests [31]. Although both culture and serology tests should be conducted for optimal diagnosis [32, 33], these have largely been superseded by polymerase chain reaction (PCR) assay [34, 35]. Nonetheless, culture and PCR lack sensitivity among those tested late after presenting with symptoms [36-39]. There is also wide variation in laboratory diagnosis of pertussis both within countries and between countries [40-42]. Since there is a lack of standardised testing for the disease we have included studies with both laboratory-confirmed and suspected/clinically diagnosed pertussis. A brief summary of the key results from each of the relevant studies is provided below, categorised where possible by comorbid condition and we have also distinguished between studies where pertussis was confirmed by laboratory testing or suspected/clinically diagnosed (e.g. diagnosed by a healthcare professional, and those studies which relied on patient self-recall of infection).

\section{Associations Between Previous Pertussis and Subsequent Development of Chronic Health Conditions/Illnesses}

\section{Asthma}

There were four studies in infants and young children that suggested previous pertussis could lead to an increased risk of developing asthma or other respiratory conditions [43-46].

The only study relying on confirmed diagnosis of pertussis was conducted in the Netherlands. It reported that those born between 1 July 2005 and 1 February 2008 with a history of laboratory-confirmed pertussis in infancy (within the first 6 months) $(n=89)$ had a relative risk of 2.8 (95\% confidence intervals [CI], 1.1-7.0) for "asthma symptoms" at toddler age (13-45 months) [46]. The small sample size in this study might, in part, explain the wide confidence intervals associated with the estimate of effect.

Four additional studies investigated this association relying on survey-based parental confirmation of prior pertussis, and found comparable results. A study conducted between December 2000 and April 2001 among children $(n=273)$ aged 6-12 years in Palestine with wheezing (including asthmatics) in the previous 


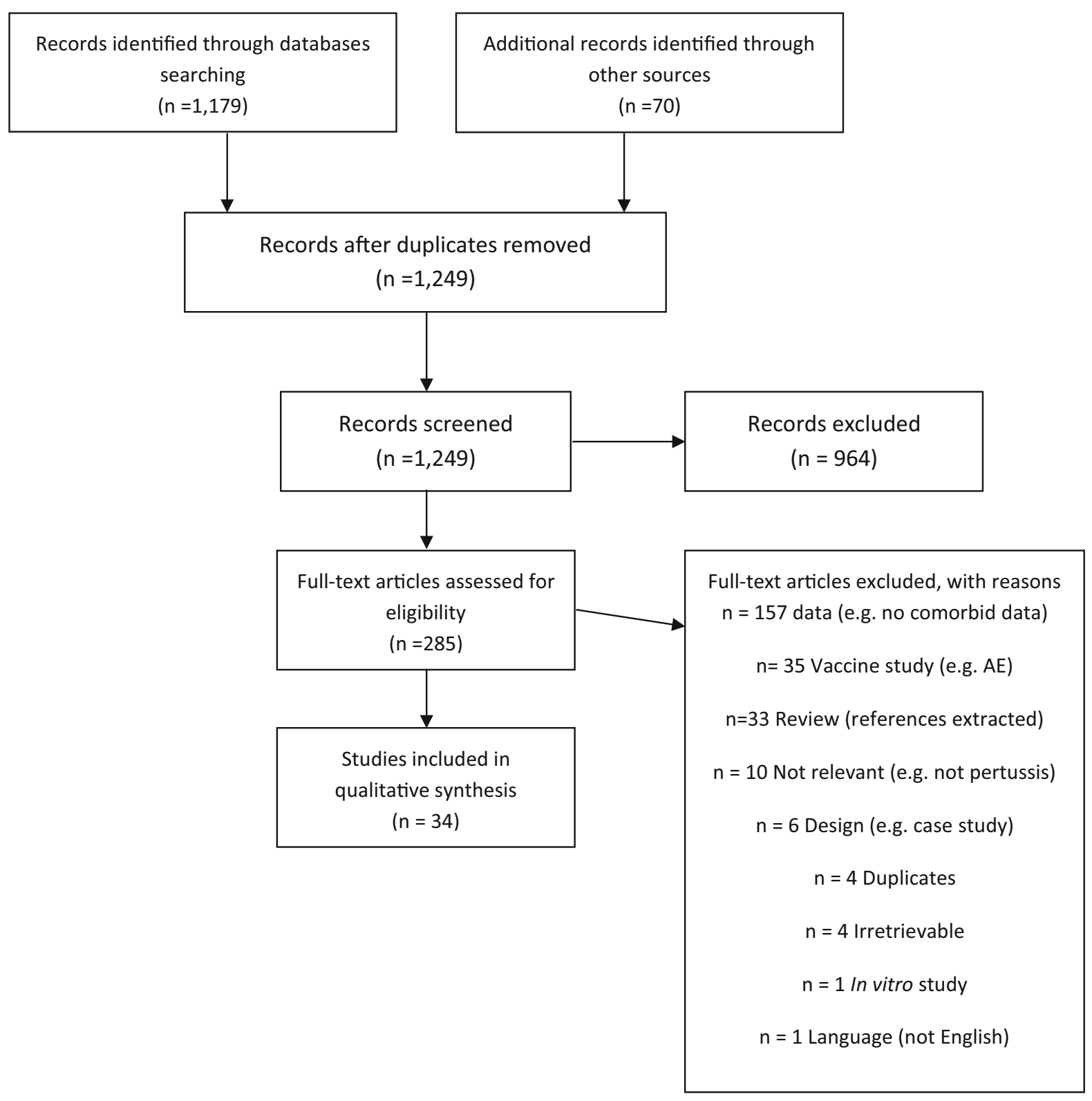

Fig. 1 PRISMA 2009 flow diagram

12 months reported that pertussis (which was reported by the parents as having occurred any time prior to the study) was an important determinant of asthma (odds ratio [OR] 4.13; 95\% CI 1.76-9.67) [43]. Another study conducted in the Middle East found that children aged 5-14 years $(n=209)$ with a history of pertussis (by self-report at any stage prior to the study) had higher odds of probable asthma (OR 3.39; 95\% CI 2.56-4.50), allergic rhinitis (OR
1.23; 95\% CI 1.06-1.42), atopic eczema (OR 1.28 ; $95 \%$ CI $1.06-0.55)$ or any of these diseases combined (OR 3.22; 95\% CI 2.41-4.32) [44]. A history of pertussis was also identified as an independent risk factor for asthma in Croatia (OR 8.14; 95\% CI 4.14-16, $P<0.001$ ) in a sample involving school children $(n=411)$ aged 6-18 years compared to non-asthmatic controls $(n=403)$ [45]. 
In contrast, one case-control study conducted in New Zealand in 1994 which included 399 children with asthma aged 7-9 years and 398 matched controls, reported no statistically significant association between pertussis infection and increased risk of asthma (OR 1.57; 95\% CI 0.58-4.24) [47].

\section{Atopic Conditions}

There were two studies examining potential associations between pertussis and atopic conditions, both of which relied upon self-report of pertussis [48, 49].

A study in the UK assessing childhood infections and atopic diseases in children $(n=2111)$ aged $10-14$ years in 1964 found that there was an increased risk of eczema in patients who reported a history of pertussis after age 3 years (OR 2.0; 95\% CI 1.2-3.5) [48]. Conversely, another study undertaken in Italy in 1987 (children [ $n=2226]$ aged 7-11 years) observed that a self-reported history of pertussis was not associated with increased risk of atopy [49]. These contradictory findings, all based on self-reporting of pertussis, give weak evidence towards such an association.

\section{Cancer}

All studies which analysed the association between pertussis and cancer relied on self-reported prior experience of pertussis. As a result, and considering the contradictory findings explained below, the level of evidence for any such association is weak.

There were two studies based on a longitudinal cohort of individuals $(n=1142)$ born in Newcastle, UK in 1947. The authors reported that childhood pertussis was associated with higher cancer mortality during age 15-60 years (adjusted hazard ratio $=4.88,95 \%$ CI 2.29-10.38) [50]. In an abstract (based on the same study), the authors reported that either tuberculosis or pertussis was independently predictive of mortality between ages 18 and 60 years [adjusted hazard ratio $(\mathrm{aHR})=2.00(95 \%$ CI 1.17-3.41) and 1.95 (95\% CI 1.21-3.14), respectively] [51]. However, the effect of pertussis on mortality was largely attributable to a higher risk of death from cancer. It was hypothesised that possibly pertussis and cancer may share some common risk factors that were not examined in the study (e.g. parental smoking or lower fruit and vegetable consumption during childhood). Other alternatives considered were poor immune function in the individuals affected, thereby increasing their susceptibility to pertussis and cancer, or that pertussis toxin itself might cause a relative increase in cell proliferation.

In contrast, three case-control studies found an inverse association between self-reported previous pertussis and a risk of developing some forms of cancers [52-54]. Becker et al., in a large multinational study $(n=12,585$ cases and 15,416 controls aged 16-96 years) found that self-reported prior pertussis disease was associated with a $15 \%$ reduced risk in the subsequent development of non-Hodgkin lymphoma (NHL) [52]. In an Italian study of 649 leukaemia cases between 1990 and 1999 (vs. 1771 controls) [53], Parodi et al. found that previous childhood pertussis was associated with lower risk of chronic lymphoid leukaemia (CLL) (OR 0.66, 95\% CI 0.45-0.98) and acute myeloid leukaemia (AML) (OR 0.52, 95\% CI 0.32-0.87). A later publication by Parodi et al. of $1193 \mathrm{NHL}$ cases, diagnosed between 1990 and 1993, found that previous childhood pertussis was associated with lower risk (OR 0.74, 95\% CI 0.62-0.88) of B cell lymphomas (1102 cases assessed vs. 1708 controls) [54].

Two case-controlled studies found no association between pertussis in childhood (either prior to age 14 [55] or age 21 [56] years) and solid tumour-related cancers $[55,56]$. Albonico et al. showed no association between previous pertussis and the risk of developing carcinoma (malignant solid epithelial tumour $[n=410]$ vs. controls $[n=379]$ ) in Switzerland between June 1993 and January 1994 [55]. Stagnaro et al. showed no association between previous pertussis and the risk of multiple myeloma (213 cases vs. 1128 controls) in Italy between 1990 and 1993 [56]. In addition, it should be noted that in both the Parodi papers described earlier $[53,54]$, there were no associations found between pertussis and some haematological cancers including chronic myeloid leukaemia and $\mathrm{T}$ cell lymphomas. 


\section{Multiple Sclerosis}

A study conducted in Switzerland compared survey data of those with multiple sclerosis $(n=606)$ in 1995 with data obtained from the Swiss Federal Health Office and from the Institute of Medical Statistics. It identified that a higher proportion of participants $(86.0 \%$ vs. $56.7 \%)$ reported prior experience of pertussis below age 10 years in those who subsequently developed multiple sclerosis [57]. However, another study reported no association (aOR 0.9, 95\% CI 0.7-1.1) between self-reported previous childhood pertussis and diagnosis of multiple sclerosis (at age $15-56$ years) in Danish cohorts born since 1940 (455 cases vs. 1801 controls) or since 1950 (182 cases vs. 690 controls) and 1975 [58]. These contradictory findings, and the more robust design of the Danish study, argue in favour of a lack of association.

\section{Epilepsy}

A Danish population-based study assessed the association between hospital-diagnosed pertussis and the long-term risk of epilepsy in a cohort born between 1978 and 2011. There were 4700 patients with pertussis diagnosis identified from the Danish National Patient Registry, of whom 90 developed epilepsy during the up to 15-year follow-up (versus 511 in the 47,000 members in the comparison cohort). Whilst the risk of epilepsy was increased in children with hospitaldiagnosed pertussis compared with the general population (hazard ratio 1.7; 95\% CI 1.3-2.1 at age 10 years), the absolute risk was low [incidence rate 1.56 per 1000 person-years (95\% CI 1.55-1.57)]. Interestingly, although the hazard ratio was not statistically different between the 1978-1997 and the 1998-2011 birth cohorts, the absolute risk of epilepsy was reduced among participants with previous pertussis diagnosis who were born from 1998 onwards and thus vaccinated with an acellular pertussis vaccine, compared to 1997 and before [59].

\section{Diabetes Mellitus}

A large longitudinal study of two British birth cohorts (the 1970 British Cohort Study [born
5-11 April 1970] and the National Child Development Study [born 3-9 March 1958]) followed from birth to ages 30 and 42 years, respectively, assessed the association of previous childhood pertussis (ascertained during periodic home interviews) and type 1 diabetes mellitus (DM) [60]. There were 100 type 1 DM cases among the 16,820 individuals assessed. Self-reported previous pertussis was associated with increased risk of type $1 \mathrm{DM}$ onset after age 10 years (aOR 2.59; 95\% CI 1.56-4.30). Of note, infant vaccination against pertussis appeared to decrease this risk (aOR 0.63; 95\% CI 0.42-0.94).

\section{Myopia}

One study assessed the association between pertussis before age 17 years and the risk of myopia among UK Biobank participants of white ethnicity aged 40-69 years who underwent autorefraction $(n=91,592) \quad$ during 2006-2010. After adjusting for potential confounding variables (age, sex, birth order and educational qualifications) a self-reported history of pertussis was associated with increased risk of myopia (OR 1.39; 95\% CI 1.03-1.87) [61].

\section{Anxiety Disorder}

A study in Germany involving 4181 adults aged 18-65 years in 1998 found that self-reported previous (during lifetime) pertussis (OR 1.69; 95\% CI 1.36-2.09) was associated with increased prevalence of any anxiety disorder and that those subjects with both infectious diseases and anxiety disorders reported lower levels of both mental and physical quality of life, compared with subjects with only one or neither condition [62].

\section{Inflammatory Bowel Disease}

No association between self-reported childhood pertussis and development of inflammatory bowel disease (Crohn's disease or ulcerative colitis) at age 10-95 years was observed in a Danish case-control study (267 cases and 267 matched control) undertaken for the period 2003-2004 (combined OR, 0.56; 95\% CI $0.27-1.20)$ [63]. 


\section{Associations Between Underlying Chronic Conditions/Illnesses and Subsequent Risk of Pertussis}

\section{Asthma}

A study in Australia (the 45 and Up Study), which included 263,094 adults aged over 45 years recruited between January 2006 and December 2008 [64], reported that after adjustment for age, sex and other factors, adults with pre-existing asthma were more likely to be diagnosed with pertussis (based on notification record) compared to those without asthma (RR 1.64; 95\% CI 1.06-2.55). Capili et al. [65] in a US-based study (223 PCR confirmed pertussis cases in 2004-2005 matched against 5537 negative controls) also reported that asthma was associated with an increased risk of pertussis (OR 1.73; 95\% CI 1.12-2.67; $P=0.013$ ), representing a $17 \%$ population attributable risk percentage of asthma for the risk of pertussis. In a Canadian study conducted by De Serres et al. during an outbreak in 1998, there was increased prevalence of asthma in patients at least 12 years old $(n=664)$ with clinical pertussis (30\% of cases laboratory confirmed), compared to the general population, but the strength of the association was not assessed. Nonetheless, while patients with asthma did not assess their pertussis illness as more severe than patients without asthma, pertussis in patients with asthma resulted in increased duration of cough $(P=0.004)$ and increased number of disturbed night sleep $(P=0.03)$ [66].

A review of pertussis cases $(n=15,942)$ with cough onset in 2011-2015 notified in the seven US Emerging Infections Program Network states found at least one underlying medical condition in $32.6 \%(168 / 515)$ of hospitalised cases. This increased to $87.2 \%(102 / 117)$ among hospitalised adults aged 21 years or older. Among adolescents and adults aged $12-20$ years, $43.5 \%$ $(10 / 23)$ had a history of asthma. More than a quarter of hospitalised adults 21-64 years old and elderly adults aged 65 years old and above with pertussis had a history of asthma $(26.3 \%$ and $26.8 \%$, respectively) [67].

A retrospective analysis of the US administrative claims databases covering the 2006-2014 period recorded a relative risk of diagnosed pertussis of 3.96 (95\% CI 3.81-4.12) in persons with pre-existing asthma (incidence rate 0.274 per 1000 person-years) compared to persons without asthma (incidence rate 0.069 per 1000 person-years) [15]. In addition, a larger proportion $(2.02 \%$ vs. $1.44 \%)$ of patients with pertussis and pre-existing asthma than patients with pertussis but no asthma had at least one pertussis-related hospitalisation in the 45 days, 3 months and 6 months period following pertussis diagnosis. Patients with pertussis and preexisting asthma accrued greater all-cause adjusted costs compared with matched patients (\$1193 more in a 45-day period, \$1301 more in a 3-month period and $\$ 1639$ more in a 6-month period; all $P<0.0001$ ).

\section{Chronic Obstructive Pulmonary Disease (COPD)}

The review of 2011-2015 pertussis cases ( $n=15,942)$ notified in the seven US Emerging Infections Program Network states described earlier in more detail also reported that $14.5 \%$ (11/76) of adults $21-64$ years old and $26.8 \%$ (11/ 41 ) of elderly adults (aged 65 years or older) had a history of COPD [67].

The retrospective analysis of US administrative claims databases (described in more detail earlier) found that the incidence of diagnosed pertussis was higher among patients with COPD ( 0.176 per 1000 person-years than among matched patients (0.069 per 1000 person-years), with a relative risk of 2.53 (95\% CI 2.40-2.68) [15]. In addition, a larger proportion (4.08\% vs. $2.33 \%$ ) of patients with pertussis and pre-existing COPD than patients with pertussis but no COPD had at least one pertussis-related hospitalisation in the 45 days, 3 and 6 months period following pertussis diagnosis. Patients with pertussis and pre-existing COPD accrued greater all-cause adjusted costs compared with matched patients ( $\$ 3694$ more in a 45 -day period, $\$ 4173$ more in a 3-month period and $\$ 6154$ more in a 6-month period; all $P<0.0001$ ).

A case-control study in Iranian adults (mean age 67.7 years) found $92.2 \%$ of 90 patients with COPD vs. $51.1 \%$ of 90 matched controls without COPD $(P<0.001)$ had serological indication (anti-PT IgG) of recent B. pertussis infection [68]. 


\section{Asthma or COPD}

A chart review survey of hospitalised laboratoryconfirmed and clinically diagnosed pertussis cases $(n=464)$ notified in 2010-2014 in the seven US Emerging Infections Program Network states identified $165(35.6 \%)$ cases with at least one underlying condition, with higher rates reported among adults aged 21 years or older (89.2\%), including a substantial proportion (31.4\%) with a history of asthma and/or COPD [69]. The authors concluded that the high rates of asthma or COPD suggest a potential risk factor for severe pertussis requiring hospitalisation, although the study did not test the hypothesis statistically.

\section{Atopic Conditions}

A population-based case-control study of pertussis cases $(n=142)$ identified in 2004 and 2005 in the USA found similar rates of atopic conditions (eczema, hay fever, atopic dermatitis and allergic rhinitis) among cases (37.4\%; 53/142) and corresponding controls (39.4\%; 112/284) [70]. The OR for atopic conditions predicting risk of pertussis was 0.91 (95\% CI 0.58-1.40), suggesting that these conditions did not increase susceptibility to pertussis.

\section{Obesity}

The previously described review of 2011-2015 pertussis cases (from seven US Emerging Infections Program Network states) found that among those hospitalised with pertussis $52.6 \%$ (40/76) of adults aged 21-64 years and 39.0\% $(16 / 41)$ aged 65 years or older were obese [67]. The Australian 45 and Up Study described earlier also examined the association between body mass index (BMI) and pertussis. Adults with a high BMI (RR 1.52 [95\% CI 1.06-2.19]; BMI $>30 \mathrm{~kg} / \mathrm{m}^{2}$ vs. BMI $<25 \mathrm{~kg} / \mathrm{m}^{2}$ ) were more likely to be notified as a pertussis case [64]. It should be noted that a subsequent analysis of data from the same cohort [71] did not observe that the risk of pertussis hospitalisation was statistically significantly higher for patients with obesity but the point estimate suggested that the risk 'may well be increased'.

\section{Miscellaneous Conditions}

The review of 2011-2015 pertussis cases (from seven US Emerging Infections Program Network states) described earlier also found other underlying conditions were common in adults aged 21-64 years $(n=76)$ and 65 years or older $(n=41)$ including having a potentially immunocompromising condition $(25 \%$ and $9.8 \%$, respectively), being diabetic ( $19.7 \%$ and $31.7 \%$ ) having some form of cardiac condition $(9.2 \%$ and $29.3 \%)$, renal disease $(10.5 \%$ and $9.8 \%)$ or a neurological condition $(5.3 \%$ and 9.8\%) [67].

A study that investigated the bacterial aetiology of community-acquired pneumonia (CAP) in hospitalised adults in Norway from May 2007 through December 2008 found that less than $1 \%(2 / 324)$ were positive for pertussis [72]. No relationship was found between CAP and pertussis infection. It was concluded that routine testing for pertussis would have no place in these groups of patients. Another study, which assessed the clinical and epidemiologic characteristics of pertussis among children aged 1-59 months hospitalised with severe pneumonia in seven African and Asian countries between August 2011 and January 2014, identified pertussis by PCR in $1.3 \%(53 / 4200)$ cases and $0.2 \%(11 / 5196)$ controls [73]. Among the African cohort, pertussis was identified in 3.7\% of 137 in-hospital deaths among those aged 1-5 months; the pertussis-infected pneumonia case fatality ratio was $12.5 \%$ (95\% CI 4.2-26.8\%; $5 / 40)$. No pertussis-positive cases aged 1-5 months were identified at the Asian sites involved. In comparison, 23\% (3/13) died among the pertussis-positive cases aged 6-59 months.

\section{Smoking}

Three studies investigated the impact of smoking on the risk and burden of pertussis. The review of 2011-2015 pertussis cases (from seven US Emerging Infections Program Network states) described earlier identified that $31.6 \%$ of adults 21 years of age and older hospitalised with pertussis were current or past smokers [67]. 
An analysis of the Australian 45 and Up Study also found that smoking was a risk factor for pertussis-related hospitalisations (aOR 2.37 [95\% CI 1.11-5.06] ever versus never) [71]. These findings were reinforced by the results of the retrospective analysis of notified pertussis cases among adolescent and adults 12 years of age and older in the 1998 outbreak in Québec, Canada. De Serres et al. found that smokers had increased duration of paroxysmal cough $(P=0.004)$, increased prevalence of sinusitis in pertussis $(P=0.008)$ and somewhat increased number of nights disturbed by coughing $(P=0.4)[66]$.

\section{Effect of Age on Pertussis Burden}

The Australian 45 and Up Study reported that the pertussis incidence in the cohort was 94 per 100,000 (95\% CI 82-108), which did not differ by age group, although hospitalisations increased with older age $(2.2,8.5$ and 13.5 per 100,000 person-years in age groups 45-64, 65-74 and 75 years or older, respectively). However, it was acknowledged that passive notification and variations in laboratory testing may have contributed to underestimation of true rates in the cohort [64]. An additional analysis [71] based upon the same study sample reinforced the conclusion that age was a significant factor in pertussis-related hospitalisations (aOR 5.4 [95\% CI 1.6-18.2] and 8.9 [95\% CI 2.3-34.7] in those aged 65-74 years and 75 years or older, respectively). The influence of age was also mentioned as a factor that appeared to increase the burden of pertussis in the analysis of cases notified during the 1998 outbreak in Québec, Canada (although in this study only $30 \%$ of cases were confirmed pertussis) [66]. The study by Mbayei et al., which looked at pertussis-related hospitalisations $(n=515)$ in the USA between 2011 and 2015, also found that patients aged 65 years or older were at increased risk of both hospitalisations and intensive care admissions (relative risk [RR], 4; 95\% CI 3-5.4 and RR, 1.8; 95\% CI 0.67-4.9) respectively, compared to other age groups [67].

\section{Impact of Pertussis on Progression of Chronic Conditions/Illnesses}

\section{Asthma}

In the review of 2011-2015 pertussis cases (from seven US Emerging Infections Program Network states) described earlier, $13.2 \%$ of $21-64$-yearolds and $24.4 \%$ of those aged 65 years or older were hospitalised with admission diagnosis of asthma or COPD exacerbation [67]. Harju et al. [74] assessed respiratory infections, including pertussis, as triggers of asthma exacerbations in Finland in 1999. Among the 103 asthmatics and 30 control subjects assessed there were 30 PCRpositive pertussis cases: five healthy controls (16.7\%), 15 mild asthmatics (28.3\%) and 10 moderate asthmatics (20\%). Pertussis-positive individuals had lower forced expiratory volume/forced vital capacity $(77.1 \%$ vs. $80.7 \%$, $P=0.012)$ and more asthma symptoms than pertussis-negative cases $(P=0.053)$ [74]. In addition, in the retrospective analysis of cases in the 1998 pertussis outbreak in Québec, Canada, 93\% of pertussis cases who were previously using bronchodilator aerosol had to increase their medication because of pertussis [66]. Finally, the retrospective analysis of US administrative claims databases (described in more detail above) found that the proportion of patients with at least one all-cause hospitalisation in the 45 days, 3 and 6 months following pertussis diagnosis was higher in the asthma cohort $(6.92 \%, 5.47 \%$ and $4.51 \%$, respectively) than in the no-asthma cohort $(2.60 \%, 2.98 \%$ and $1.92 \%$, respectively) [15].

\section{COPD}

As noted earlier, both the retrospective analysis of 2011-2015 notified cases in the seven US Emerging Infections Program Network states and the review of cases notified in the 1998 outbreak in Québec, Canada identified significant increase in signs of asthma or COPD exacerbation among pertussis cases [66, 67]. In addition, as for asthma, the retrospective analysis of US administrative claims databases 
(described in more detail earlier) found that the proportion of patients with at least one all-cause hospitalisation in the 45 days, 3 and 6 months following pertussis diagnosis was higher in the COPD cohort $(12.24 \%, 10.21 \%$ and $8.46 \%$, respectively) than in the non-COPD cohort (6.41\%, 5.54\% and 4.95\%, respectively) [15].

Although the case-control study undertaken in Iran among patients with COPD and controls without COPD found increased signs of pertussis infection among patients with COPD, no association was observed between $B$. pertussis infection and severity of COPD [68].

A small study of adults $(n=26)$ with acute exacerbations of chronic bronchitis conducted in Switzerland between 2000 and 2002 [75] identified B. pertussis infection by serology in eight $(31 \%)$ of the patients. The study showed that pertussis infection was associated with a shorter duration of cough (mean 15 vs. 41 days, $P=0.04$ ) compared with cough of unknown aetiology. Cough of at least 21 days duration was present in three (43\%) of seven patients with evidence of Bordetella infection compared to $17(94 \%)$ of 18 controls $(P=0.012)$. The shorter duration of cough in those with pertussis was somewhat unexpected and it was postulated that this was probably due to pertussis reinfection, which is common in adults, leading to comparatively mild symptoms in these cases owing to the partial immunity from previous infection.

\section{DISCUSSION}

Over time, a significant amount of evidence has been accumulated on the potential association of pertussis with various chronic conditions, resulting in heterogeneous findings, but also some clear signs of correlation, specifically with chronic respiratory diseases.

Pertussis infection induces strong immunomodulatory mechanisms which are known to play an important role in the pathophysiology of whooping cough. Therefore, it stands to reason that these actions on the immune system may play a role in triggering or enhancing other pathologies which may involve the immune system. The evidence we uncovered on the possible associations of pertussis with the subsequent development of chronic conditions is generally weak. One of its main shortcomings is that given the time lag between pertussis and the condition of interest, evidence of prior pertussis infection often relied on self-reporting. The fundamental bias introduced by such an exposure definition puts into question the validity of the findings. Nonetheless, in the case of asthma, one study, albeit of small size, using confirmed cases of pertussis demonstrated an association between infant pertussis and subsequent development of asthma symptoms in childhood [46]. While the rest of the evidence on risk of asthma after pertussis infection relied on self-reported cases of pertussis, it was generally consistent with this finding [43-45]. The immunologic nature of asthma may be at play in this association, and it seems reasonable to think that the immunomodulatory effects of pertussis, particularly if effected on a specifically susceptible background, may stimulate the development of allergic asthma in subsequent years. The results presented here are, in many ways, consistent with the hypotheses of Rubin and Glazer (2018) [76] who suggested that $B$. pertussis colonization was an important cause of asthma and diseases of allergic sensitization after applying the Bradford Hill criteria in a review of data from the USA and the former Democratic Republic of Germany (1980-2007). It has also been suggested, in contrast, that previous infections with pertussis, early in childhood, promote allergic sensitisation [44, 49, 47, 74, 48, 60, 75]. Similar hypotheses have been made for the association found between pertussis and diabetes, especially type 1 diabetes. Although relying on self-reported pertussis, one study did conclude to an increased risk of diabetes following a pertussis episode, and further supported this with indications that pertussis vaccination reduced this risk [60]. The authors in this case suggested that the immunomodulatory effect of pertussis may trigger autoimmune mechanisms causing type 1 diabetes. With somewhat different mechanisms at play, one study relying on diagnosed pertussis cases found a significant association between infant pertussis and later risk of epilepsy [59]. While 
the overall risk was low, the authors hypothesised that neurological damage which can result from severe pertussis disease in early infancy (cases in this study were hospital diagnosed) could be behind this association [59]. In fact, an apparent reduction in incidence of epilepsy diagnosis noted in this study coincided with the introduction of the more immunogenic acellular pertussis vaccine, and possibly reflected the milder course of pertussis in breakthrough pertussis cases. For the other conditions studied, including atopic conditions [49], cancers [52-55], multiple sclerosis [57, 58], myopia [61] and inflammatory bowel disease [63], the evidence we found was weak (i.e. relying on selfreported pertussis, single studies, small sample sizes or design limitations) and often contradictory, making it difficult to draw conclusions.

Conversely, a larger and more robust body of evidence exists to show that underlying conditions/illnesses could increase the likelihood of developing pertussis or more severe forms of pertussis, potentially requiring medical interventions and, in some cases, hospitalisation. In particular, consistent evidence from different settings (i.e. Australia [64, 71], Canada [66], Iran [68] and mainly the USA $[15,65,67,69])$ and using different data sources or methodologies (i.e. cohorts, notification databases, administrative claims databases) indicates that asthma and/or COPD [15, 64-67, 69] is particularly associated with a 1.64-fold [64] to almost 4-fold [43] increased likelihood of pertussis. Asthma represented an associated risk factor in $43.5 \%$ of adolescents hospitalised with pertussis, and asthma or COPD was a risk factor in more than a quarter of adults hospitalised with pertussis [67]. These conditions appear not only to increase the risk of pertussis but also to increase the duration and severity of its symptoms, the risk of hospitalisation for pertussis and consequently the costs associated with pertussis in these age groups [15]. The inflammatory pathophysiology of pertussis and the offense caused by pertussis infection on reactive airways of patients with asthma and COPD provide the likely explanation for this increased burden of whooping cough in these specific populations. In contrast, there was no association found between atopic conditions and subsequent pertussis [70]. Studies in Australia and the USA found an association between obesity and increased risk of pertussis diagnosis [64, 67]. However, the association is not clearly explained. In fact, it is unclear whether obesity represents a risk factor for pertussis per se, or whether it is a proxy for other associated comorbidities which could more clearly provide a causal risk factor for pertussis. Interestingly, several studies also explored the role of smoking and age $[66,67,71]$ in the risk of pertussis and found that current or past smoking, as well as older age, increased the risk and severity of pertussis. In the case of smoking, the damage to the airways caused by tobacco smoke, and the increased risk of COPD in smokers, could certainly explain on their own the increased risk of pertussis. The effect of age likely reflects the higher likelihood of clinical pertussis and more severe forms of pertussis that come from the combined effect of more fragile health resulting from aging and the increased prevalence of chronic diseases. Other studies also investigated and suggested how other conditions, including compromised immune systems [67], diabetes [60] and cardiovascular disease [67], could increase the risk of pertussis. The evidence is weaker for these conditions and it is possible that pertussis infection on such backgrounds may lead to higher likelihood of diagnosis and healthcare usage.

Finally, the evidence we found also indicates that, in the presence of an underlying chronic conditions/illnesses, pertussis can lead to exacerbation of the underlying comorbidity and result in increased healthcare need. Consistent and strong study results from the USA [15, 65, 67, 69], Canada [66], Finland [74] and Switzerland [75] using diverse data sources and analytical methods showed that pertussis infection in persons with asthma or COPD leads to poorer respiratory capacity, more and stronger asthma/COPD exacerbations, increased use of bronchodilator and increased hospitalisation, resulting in higher healthcare costs $[69,74]$.

The principal strength of our study is that it uses a well-defined and accepted methodology (i.e. that of a systematic review) to identify suitable studies for inclusion. The weakness of our study is that we restricted the literature to 
those publications which are in the English language, so we may have omitted studies published in other languages, and by restricting our search to three databases we may have been unable to identify studies which were published in non-indexed journals. In addition, much of the data were from single studies of a condition, which make interpretation of the data difficult. Whilst some of the studies were large and longitudinal, many were hampered by the lack of definitive laboratory confirmation of pertussis. As noted above, the diagnosis of pertussis in adults is often not reliant upon laboratory testing which limits the robustness of the evidence suggesting potential associations or lack thereof.

\section{CONCLUSION}

Previous pertussis appears to contribute to the increased likelihood of developing some respiratory conditions like asthma, and conversely those with asthma or COPD are at increased risk of pertussis and severe pertussis requiring further intervention. Further research is required to better confirm and characterise these associations, and the pathophysiological mechanisms behind the potential associations with pertussis.

\section{ACKNOWLEDGEMENTS}

Funding. Sponsorship for this study and the rapid service fees for the journal were funded by Sanofi Pasteur, France.

Editorial Assistance. The authors acknowledge Richard Glover of inScience Communications, Springer Healthcare Ltd, Chester, UK for editorial assistance with the preparation of this manuscript. This assistance was funded by Sanofi Pasteur. The authors also thank Burnedette Rose-Hill for editorial assistance and manuscript coordination on behalf of Sanofi Pasteur.

Authorship. All named authors meet the International Committee of Medical Journal
Editors (ICMJE) criteria for authorship for this article, take responsibility for the integrity of the work as a whole, and have given their approval for this version to be published.

Authorship Contributions. Denis MacinaConceptualization, methodology, investigation, Writing-review and editing. Keith Evans-Methodology, investigation, Writingreview and editing.

Disclosures. Denis Macina is an employee of Sanofi Pasteur. Keith Evans was provided with funding by Sanofi Pasteur to conduct the literature review in collaboration with Denis Macina.

Compliance with Ethics Guidelines. This article is based on previously conducted studies and does not contain any studies with human participants or animals performed by any of the authors.

Data Availability. Data sharing is not applicable to this article as no datasets were generated or analysed during the current study.

Open Access. This article is licensed under a Creative Commons Attribution-NonCommercial 4.0 International License, which permits any non-commercial use, sharing, adaptation, distribution and reproduction in any medium or format, as long as you give appropriate credit to the original author(s) and the source, provide a link to the Creative Commons licence, and indicate if changes were made. The images or other third party material in this article are included in the article's Creative Commons licence, unless indicated otherwise in a credit line to the material. If material is not included in the article's Creative Commons licence and your intended use is not permitted by statutory regulation or exceeds the permitted use, you will need to obtain permission directly from the copyright holder. To view a copy of this licence, visit http://creativecommons.org/licenses/bync/4.0/. 


\section{REFERENCES}

1. World Health Organization. Pertussis. 2018. https://www.who.int/immunization/diseases/ pertussis/en/. Accessed 26 May 2020.

2. Cho BH, Acosta AM, Leidner AJ, Faulkner AE, Zhou F. Tetanus, diphtheria and acellular pertussis (Tdap) vaccine for prevention of pertussis among adults aged 19 years and older in the United States: a costeffectiveness analysis. Prev Med. 2020;134:106066.

3. Fedele G, Spensieri F, Palazzo R, et al. Bordetella pertussis commits human dendritic cells to promote a Th1/Th17 response through the activity of adenylate cyclase toxin and MAPK-pathways. PLoS ONE. 2010;5(1):e8734.

4. Fennelly NK, Sisti F, Higgins SC, et al. Bordetella pertussis expresses a functional type III secretion system that subverts protective innate and adaptive immune responses. Infect Immun. 2008;76(3): 1257-66.

5. Hickey FB, Brereton CF, Mills KH. Adenylate cycalse toxin of Bordetella pertussis inhibits TLR-induced IRF-1 and IRF-8 activation and IL-12 production and enhances IL-10 through MAPK activation in dendritic cells. J Leukoc Biol. 2008;84(1):234-43.

6. Ostolaza H, Martin C, Gonzalez-Bullon D, Uribe KB, Etxaniz A. Understanding the mechanism of translocation of adenylate cyclase toxin across biological membranes. Toxins (Basel). 2017;9(10): 295.

7. Paccani SR, Finetti F, Davi M, et al. The Bordetella pertussis adenylate cyclase toxin binds to $\mathrm{T}$ cells via LFA-1 and induces its disengagement from the immune synapse. J Exp Med. 2011;208(6):1317-30.

8. Spensieri F, Fedele G, Fazio C, et al. Bordetella pertussis inhibition of interleukin-12 (IL-12) p70 in human monocyte-derived dendritic cells blocks IL12 p35 through adenylate cyclase toxin-dependent cyclic AMP induction. Infect Immun. 2006;74(5): 2831-8.

9. Ajdacic-Gross V, Mutsch M, Rodgers S, et al. A step beyond the hygiene hypothesis-immune-mediated classes determined in a population-based study. BMC Med. 2019;17(1):75.

10. Ennis DP, Cassidy JP, Mahon BP. Whole-cell pertussis vaccine protects against Bordetella pertussis exacerbation of allergic asthma. Immunol Lett. 2005;97(1):91-100.

11. Lamberti Y, Gorgojo J, Massillo C, Rodriguez ME. Bordetella pertussis entry into respiratory epithelial cells and intracellular survival. Pathog Dis. 2013;69(3):194-204.

12. Farooqi IS, Hopkin JM. Early childhood infection and atopic disorder. Thorax. 1998;53(11):927-32.

13. Kandeil W, Atanasov P, Avramioti D, Fu J, Demarteau N, Li X. The burden of pertussis in older adults: what is the role of vaccination? A systematic literature review. Expert Rev Vaccines. 2019;18(5): 439-55.

14. Wateska AR, Nowalk MP, Zimmerman RK, Smith KJ, Lin CJ. Cost-effectiveness of increasing vaccination in high-risk adults aged 18-64 years: a model-based decision analysis. BMC Infect Dis. 2018;18(1):52.

15. Buck PO, Meyers JL, Gordon LD, Parikh R, Kurosky SK, Davis KL. Economic burden of diagnosed pertussis among individuals with asthma or chronic obstructive pulmonary disease in the USA: an analysis of administrative claims. Epidemiol Infect. 2017;145(10):2109-21.

16. Beaver ME, Karow CM. Incidence of seropositivity to Bordetella pertussis and mycoplasma pneumoniae infection in patients with chronic laryngotracheitis. Laryngoscope. 2009;119(9):1839-43.

17. Esposito S, Franco E, Gavazzi G, et al. The public health value of vaccination for seniors in Europe. Vaccine. 2018;36(19):2523-8.

18. Tong J, Buikema A, Horstman T. Epidemiology and disease burden of pertussis in the United States among individuals aged 0-64 over a 10-year period (2006-2015). Curr Med Res Opin. 2020;36(1): $127-37$.

19. van der Maas NA, Mooi FR, de Greeff SC, Berbers GA, Spaendonck MA, de Melker HE. Pertussis in the Netherlands, is the current vaccination strategy sufficient to reduce disease burden in young infants? Vaccine. 2013;31(41):4541-7.

20. Ridda I, Yin JK, King C, Raina MacIntyre C, McIntyre P. The importance of pertussis in older adults: a growing case for reviewing vaccination strategy in the elderly. Vaccine. 2012;30(48):6745-52.

21. Riffelmann M, Littmann M, Hulsse C, O'Brien J, Wirsing von Konig CH. Pertussis: incidence, symptoms and costs. Dtsch Med Wochenschr. 2006;131(50):2829-34.

22. Caro JJ, Getsios D, Payne K, Annemans L, Neumann PJ, Trindade E. Economic burden of pertussis and the impact of immunization. Pediatr Infect Dis J. 2005;24(5 Suppl):S48-54. 
23. McGuiness CB, Hill J, Fonseca E, Hess G, Hitchcock W, Krishnarajah G. The disease burden of pertussis in adults 50 years old and older in the United States: a retrospective study. BMC Infect Dis. 2013;23(13): 32.

24. Rothstein E, Edwards K. Health burden of pertussis in adolescents and adults. Pediatr Infect Dis J. 2005;24(5 Suppl):S44-7.

25. van Hoek AJ, Campbell H, Andrews N, Vasconcelos M, Amirthalingam G, Miller E. The burden of disease and health care use among pertussis cases in school aged children and adults in England and Wales; a patient survey. PLoS ONE. 2014;9(11): e111807.

26. Jenkins VA, Savic M, Kandeil W. Pertussis in highrisk groups: an overview of the past quarter-century. Hum Vaccin Immunother. 2020;16:1-9.

27. Bero L, Chartres N, Diong J, et al. The risk of bias in observational studies of exposures (ROBINS-E) tool: concerns arising from application to observational studies of exposures. Syst Rev. 2018;7(1):242.

28. Schünemann $H$, Brożek J, Guyatt $G$, Oxman A. Handbook for grading the quality of evidence and the strength of recommendations using the GRADE approach. 2013. https://gdt.gradepro.org/app/ handbook/handbook.html\#h.dce0ghnajwsm. Accessed 1 Feb 2020.

29. Viljanen MK, Ruuskanen O, Granberg C, Salmi TT. Serological diagnosis of pertussis: IgM, IgA and IgG antibodies against Bordetella pertussis measured by enzyme-linked immunosorbent assay (ELISA). Scand J Infect Dis. 1982;14(2):117-22.

30. Lawrence AJ, Paton JC. Efficacy of enzyme-linked immunosorbent assay for rapid diagnosis of Bordetella pertussis infection. J Clin Microbiol. 1987;25(11):2102-4.

31. Campbell PB, Masters PL, Rohwedder E. Whooping cough diagnosis: a clinical evaluation of complementing culture and immunofluorescence with enzyme-linked immunosorbent assay of pertussis immunoglobulin A in nasopharyngeal secretions. J Med Microbiol. 1988;27(4):247-54.

32. Halperin SA, Bortolussi R, Wort AJ. Evaluation of culture, immunofluorescence, and serology for the diagnosis of pertussis. J Clin Microbiol. 1989;27(4): 752-7.

33. Mertsola J, Ruuskanen O, Kuronen T, Meurman O, Viljanen MK. Serologic diagnosis of pertussis: evaluation of pertussis toxin and other antigens in enzyme-linked immunosorbent assay. J Infect Dis. 1990;161(5):966-71.
34. He Q, Mertsola J, Soini H, Skurnik M, Ruuskanen O, Viljanen MK. Comparison of polymerase chain reaction with culture and enzyme immunoassay for diagnosis of pertussis. J Clin Microbiol. 1993;31(3): $642-5$.

35. Chia JH, Su LH, Lin PY, et al. Comparison of multiplex polymerase chain reaction, culture, and serology for the diagnosis of Bordetella pertussis infection. Chang Gung Med J. 2004;27(6):408-15.

36. Bock JM, Burtis CC, Poetker DM, Blumin JH, Frank MO. Serum immunoglobulin $\mathrm{G}$ analysis to establish a delayed diagnosis of chronic cough due to Bordetella pertussis. Otolaryngol Head Neck Surg. 2012;146(1):63-7.

37. Beaman $\mathrm{MH}$, Karimi M, Hodge M, Keil AD, Campbell P. Diagnosis of pertussis using nasopharyngeal $\operatorname{IgA}$ and polymerase chain reaction in specimens from outpatients in Australia. Eur J Microbiol Immunol (Bp). 2014;4(4):177-83.

38. Lee AD, Cassiday PK, Pawloski LC, et al. Clinical evaluation and validation of laboratory methods for the diagnosis of Bordetella pertussis infection: culture, polymerase chain reaction (PCR) and antipertussis toxin IgG serology (IgG-PT). PLoS ONE. 2018;13(4):e0195979.

39. Tozzi AE, Celentano LP, Ciofi degli Atti ML, Salmaso S. Diagnosis and management of pertussis. CMAJ. 2005;172(4):509-15.

40. Dalby T, Fry NK, Krogfelt KA, Jensen JS, He Q, pertussis EQA participants. Evaluation of PCR methods for the diagnosis of pertussis by the European surveillance network for vaccine-preventable diseases (EUVACNET). Eur J Clin Microbiol Infect Dis. 2013;32(10):1285-9.

41. Strebel PM, Cochi SL, Farizo KM, Payne BJ, Hanauer SD, Baughman AL. Pertussis in Missouri: evaluation of nasopharyngeal culture, direct fluorescent antibody testing, and clinical case definitions in the diagnosis of pertussis. Clin Infect Dis. 1993;16(2): 276-85.

42. Waggoner-Fountain L, Hayden GF. Pertussis in primary care practice. Recent advances in diagnosis, treatment, and prevention. Prim Care. 1996;23(4): 793-804.

43. El-Sharif N, Abdeen Z, Barghuthy F, Nemery B. Familial and environmental determinants for wheezing and asthma in a case-control study of school children in Palestine. Clin Exp Allergy. 2003;33(2):176-86.

44. Waked M, Salameh P. Risk factors for asthma and allergic diseases in school children across Lebanon. J Asthma Allergy. 2009;2009(2):1-7. 
45. Aberle N, Kljaic Bukvic B, Blekic M, et al. Risk factors for childhood asthma in Croatia. Allergy Eur J Allergy Clin Immunol. 2010;65:314.

46. De Greeff SC, Van Buul LW, Westerhof A, et al. Pertussis in infancy and the association with respiratory and cognitive disorders at toddler age. Vaccine. 2011;29(46):8275-8.

47. Wickens KL, Crane J, Kemp TJ, et al. Family size, infections, and asthma prevalence in New Zealand children. Epidemiology. 1999;10(6):699-705.

48. Bodner C, Godden D, Seaton A. Family size, childhood infections and atopic diseases. The Aberdeen WHEASE Group. Thorax. 1998;53(1):28-32.

49. Forastiere F, Agabiti N, Corbo GM, et al. Socioeconomic status, number of siblings, and respiratory infections in early life as determinants of atopy in children. Epidemiology. 1997;8(5):566-70.

50. Tennant PWG, Parker L, Thomas JE, Craft SAW, Pearce MS. Childhood infectious disease and premature death from cancer: a prospective cohort study. Eur J Epidemiol. 2013;28(3):257-65.

51. Tennant PWG, Parker L, Pearce MS. Childhood infectious diseases and premature adult mortality: results from the Newcastle thousand families study. Am J Epidemiol. 2011;173:S26.

52. Becker N, Falster MO, Vajdic CM, et al. Self-reported history of infections and the risk of non-Hodgkin lymphoma: an InterLymph pooled analysis. Int J Cancer. 2012;131(10):2342-8.

53. Parodi S, Crosignani P, Miligi L, et al. Childhood infectious diseases and risk of leukaemia in an adult population. Int J Cancer. 2013;133(8):1892-9.

54. Parodi S, Seniori Costantini A, Crosignani P, et al. Childhood infectious diseases and risk of nonHodgkin's lymphoma according to the WHO classification: a reanalysis of the Italian multicenter case-control study. Int J Cancer. 2019;146:977-86.

55. Albonico HU, Braker HU, Husler J. Febrile infectious childhood diseases in the history of cancer patients and matched controls. Med Hypotheses. 1998;51(4):315-20.

56. Stagnaro E, Parodi S, Costantini AS, et al. Childhood infectious diseases and risk of multiple myeloma: an analysis of the Italian multicentre case-control study. Epidemiol Infect. 2018;146(12): $1572-4$.

57. Bachmann S, Kesselring J. Multiple sclerosis and infectious childhood diseases. Neuroepidemiology. 1998;17(3):154-60.
58. Bager P, Nielsen NM, Bihrmann K, et al. Childhood infections and risk of multiple sclerosis. Brain. 2004;127(Part 11):2491-7.

59. Olsen M, Thygesen SK, Østergaard JR, et al. Hospital-diagnosed pertussis infection in children and long-term risk of epilepsy. JAMA. 2015;314(17): 1844-9.

60. Montgomery SM, Ehlin AGC, Ekbom A, Wakefield AJ. Pertussis infection in childhood and subsequent type 1 diabetes mellitus. Diabet Med. 2002;19(12): 986-93.

61. Guggenheim JA, Williams C. Childhood febrile illness and the risk of myopia in UK Biobank participants. Eye (Basingstoke). 2016;30(4):608-14.

62. Witthauer C, Gloster AT, Meyer AH, Goodwin RD, Lieb R. Comorbidity of infectious diseases and anxiety disorders in adults and its association with quality of life: a community study. Front Public Health. 2014;2:80.

63. Hansen TS, Jess T, Vind I, et al. Environmental factors in inflammatory bowel disease: a case-control study based on a Danish inception cohort. J Crohns Colitis. 2011;5(6):577-84.

64. Liu BC, McIntyre P, Kaldor JM, Quinn HE, Ridda I, Banks E. Pertussis in older adults: prospective study of risk factors and morbidity. Clin Infect Dis. 2012;55(11):1450-6.

65. Capili CR, Hettinger A, Rigelman-Hedberg N, et al. Increased risk of pertussis in patients with asthma. J Allergy Clin Immunol. 2012;129(4):957-63.

66. De Serres G, Shadmani R, Duval B, et al. Morbidity of pertussis in adolescents and adults. J Infect Dis. 2000;182(1):174-9.

67. Mbayei SA, Faulkner A, Miner C, et al. Severe pertussis infections in the United States, 2011-2015. Clin Infect Dis. 2019;69(2):218-26.

68. Hashemi SH, Nadi E, Hajilooi M, Seif-Rabiei MA, Samaei A. High seroprevalence of Bordetella pertussis in patients with chronic obstructive pulmonary disease: a case-control study. Tanaffos. 2015;14(3): 172-6.

69. Meyer S, Faulkner A, Miner C, et al. Pertussis infections in hospitalized patients-United States, 2010-2014. Open Forum Infect Dis. 2016;2016:3.

70. Rigelman-Hedberg NE, Hettinger AS, Fink LR, et al. Assessment of susceptibility to pertussis in individuals with atopic conditions other than asthma. J Allergy Clin Immunol. 2009;123(2):S162. 
71. Karki S, McIntyre P, Newall AT, MacIntyre CR, Banks E, Liu B. Risk factors for pertussis hospitalizations in Australians aged 45 years and over: a population based nested case-control study. Vaccine. 2015;33(42):5647-53.

72. Røysted W, Simonsen $\varnothing$, Jenkins A, et al. Aetiology and risk factors of community-acquired pneumonia in hospitalized patients in Norway. Clin Respir J. 2016;10(6):756-64.

73. Barger-Kamate B, Knoll MD, Kagucia EW, et al. Pertussis-associated pneumonia in infants and children from low-and middle-income countries participating in the PERCH study. Clin Infect Dis. 2016;63:S187-96.
74. Harju $\mathrm{TH}$, Leinonen $\mathrm{M}$, Nokso-Koivisto $\mathrm{J}$, et al. Pathogenic bacteria and viruses in induced sputum or pharyngeal secretions of adults with stable asthma. Thorax. 2006;61(7):579-84.

75. Bonhoeffer J, Bar G, Riffelmann M, Soler M, Heininger $U$. The role of Bordetella infections in patients with acute exacerbation of chronic bronchitis. Infection. 2005;33(1):13-7.

76. Rubin K, Glazer S. The pertussis hypothesis: Bordetella pertussis colonization in the etiology of asthma and diseases of allergic sensitization. Med Hypotheses. 2018;2018(120):101-15. 